Article

\title{
Optimization Strategy of Electric Vehicles Charging Path Based on "Traffic-Price-Distribution" Mode
}

\author{
Wanhao Yang ${ }^{1}$, Hong Wang ${ }^{2}$, Zhijie Wang ${ }^{1, *}$, Xiaolin Fu ${ }^{1}$, Pengchi Ma ${ }^{1}$, Zhengchen Deng ${ }^{3}$ \\ and Zihao Yang 4 \\ 1 College of Electrical Engineering, Shanghai Dianji University, Shanghai 201306, China; \\ 186001010415@st.sdju.edu.cn (W.Y.); 186001010319@st.sdju.edu.cn (X.F.); 186001010408@st.sdju.edu.cn (P.M.) \\ 2 School of Economics \& Management, Tongji University, Shanghai 200092, China; wanghongpy@163.com \\ 3 College of Electrical Engineering, Shanghai University of Electric Power, Shanghai 200090, China; \\ dengzhengchen@163.com \\ 4 College of Electrical and Electronic Engineering, North China Electric Power University, Beijing 102206, \\ China; ziihaoo@163.com \\ * Correspondence: wangzj@sdju.edu.cn; Tel.: +86-189-6458-6826
}

Received: 29 November 2019; Accepted: 27 February 2020; Published: 20 June 2020

\begin{abstract}
According to the current optimization problem of electric vehicle charging path planning, a charging path optimization strategy for electric vehicles is proposed, which is under the "traffic-price-distribution" mode. Moreover, this strategy builds an electric vehicle charging and navigation system on the basis of the road traffic network model, real-time electricity price model and distribution network model. Based on the Dijkstra shortest path algorithm and Monte Carlo time-space prediction method, it gets the optimal charging path navigation with the goal of minimizing the charging cost of electric vehicles. The simulation results in MATLAB and MATPOWER (MATLAB R2018a, MATPOWER3.1b2, PSERC, Cannell University) show that the electric vehicle charging path optimization strategy can solve the local traffic congestion problem better and improve the safety and stability of the distribution network because of the fully considering the convenience of electric vehicle charging.
\end{abstract}

Keywords: electric vehicles charging navigation system; charging path; road transportation network; distribution network; real-time electricity price

\section{Introduction}

The World Oil Outlook 2017 claimed that the transport sector accounted for $54.6 \%$ of world oil consumption in 2017 [1]. According to this report, this sector will consume $64.9 \%$ of the world's oil consumption by 2040. With the development of industrialization, the global awareness of the energy crisis is increasing. Compared with the current depletion of traditional petroleum resources, electric vehicles (EVs), which are treated as a low-carbon green vehicle driven by electricity, undoubtedly could offer energy conservation and emission reduction in the future, and these should be research goals.

As a timesaving and efficient charging method, fast charging is becoming more and more popular among users as electric vehicles become popular. Affected by electric vehicle travel characteristics and road structure, the fast charging demand for electric vehicles has some obvious spatiotemporal distribution characteristics. At present, the layout of China's fast-charging stations does not match the distribution of charging demand. Therefore, problems such as uneven utilization of fast-charging stations, long vehicle queuing time, and large voltage shifts in the distribution network need to be resolved [2].

According to the Global EV Outlook [3], the number of EVs worldwide could exceed 130 million by 2030 under the assumption that government policies continue supporting the expansion of 
electromobility. Due to the unique charging characteristics of electric vehicles, the charging behavior of large-scale electric vehicles not only puts pressure on the transportation system but also affects the safe and stable operation of the distribution network [4-6].

Spitzer, M. researched on optimized integration of electric vehicles in low-voltage distribution grids and analyzed the impact of uncoordinated charging, as well as optimization-based coordination strategies on the voltage stability and phase unbalances of a representative European semi-urban low voltage grid, and solved the phase imbalance problem of distribution networks [7]. Two papers [8,9] also proposed a good strategy to reduce phase imbalance; Jabalameli proposed charge/discharge strategies for plug-in electric vehicles in unbalanced active power distribution networks with reactive power compensation to compensate for phase imbalance in the distribution network through reactive power compensation; and found single-phase residential charging can also initiate or contribute to voltage unbalance conditions in the distribution networks [8]. Muratori focuses on the impact of plug-in electric vehicle (PEV) charging behavior on residential power demand and uses highly analyzed residential electricity demand models and PEV usage to assess the impact of uncoordinated household PEV charging on residential electricity consumption [9]; Diaz-Londono proposed a charging strategy to improve the flexibility of electric vehicle charging stations [10]. By providing specific definitions of the flexibility of charging stations, two optimal charging strategies were given to achieve the purpose of reducing the operating costs of charging stations. Among them, two studies $[7,8]$ also discussed the impact of price on grid stability. [7] considered the actual arrival and departure time of electric vehicles and provided a coordinated charging strategy to optimize the distribution network. [8] improved the node voltage curve by providing reactive power injection of PEV inverters on selected nodes based on variable pricing/contract schemes.

At present, research on the charging path planning of large-scale electric vehicles based on traffic conditions and distribution network conditions is still very rare. Kobayashi Yuichi identified an electric vehicle charging path planning method based on the starting and ending traffic locations of an electric vehicle and actual traffic congestion [11]. Guo proposed "electrical distance" based on traffic simulation; established a path optimization framework that integrates transportation systems and charging stations. The current stimulus for orderly charging is mostly electricity prices and charging time [12]. Bayram introduced a game theory based on decentralization to control the formulation of short-term dynamic electricity prices and to guide users to actively participate in the selection. Thereby it can adjust the load balance of charging stations [13]. Xu proposed a hierarchical regional charging control framework that can meet the user's charging needs and has some effects on reducing the distribution network peak loads and charging station operating costs [14]. With the development of intelligent communication technology (ICT) and intelligent transportation system (ITS) technology, the real-time information sharing among electric vehicles, fast-charging stations and distribution networks has become possible. Existing vehicle management systems provided services for the interaction among electric vehicles, ITS, and fast-charging stations in a binary file format through Hypertext Transfer Protocol (HTTP), forming a generalized intelligent system among electric vehicles, fast-charging stations, and distribution networks to complete the electric vehicle charging guide [15].

It is not difficult to find that none of the above papers further studied the comprehensive impact of real-time electricity price mechanisms on the transportation network, distribution network, and user charging behavior. Therefore, based on the previous research [16,17], Yan and others put forward the idea of combining electric vehicles, distribution networks, and transportation networks into a "vehicle-network-road" system, and initially discussed the optimal charging path planning technology for electric vehicles [16]. Shao further analyzed the spatiotemporal prediction of the charging load of large-scale electric vehicles under the "vehicle-network-road" model and its impact on the power distribution flows [17]. This paper develops a more comprehensive real-time electricity price mechanism, and builds a "traffic-price-distribution" model through MATLAB simulation technology, and proposes a method that not only fully considers the information exchange situation of the road traffics network and distribution network, also implements a convenient path optimization strategy for 
electric vehicle charging. The optimal charging path recommended by the optimization strategy can better solve the problems of traffic congestion, uneven load distribution of charging stations, and safety of the distribution network operation while reducing the charging cost of large-scale electric vehicle groups. The main innovations of this paper can be summarized as follows:

1. This paper proposes an optimization strategy for planning the optimal charging path of electric vehicles based on the "traffic-price-distribution" model; and constructs a new electric vehicle path navigation system. Based on the Dijkstra shortest path algorithm and Monte Carlo spatiotemporal prediction method, the charging simulation process for 40,000 electric vehicles is completed. Adopting the optimal charging path navigation, you can more rationally plan the charging load between charging stations, avoid the phenomenon of partial overloading with the charging stations as much as possible, effectively improve the charging efficiency of electric vehicles, and greatly reduce the charging time of electric vehicles.

2. Adopting the optimal charging path navigation method can effectively alleviate the local traffic congestion caused by the accumulation of more electric vehicles at a charging station and improve the operational status of the entire transportation network.

3. This paper introduces the real-time electricity price mechanism, considering the vital interests in electric vehicle users, and more reasonably saving charging costs for electric vehicle users.

\section{Traffic-Price-Distribution Model}

\subsection{Road Traffic Network Model}

Based on the previous researches, this paper builds a "road traffics network-real-time electricity price-distribution networks" model and researches the optimal path navigation of electric vehicles. It is assumed that all-electric vehicles adopt the form of fast charging, and its unique charging characteristics and space characteristics have a certain impact on the road transportation network and distribution network. The relationship between the two is shown in Figure 1.

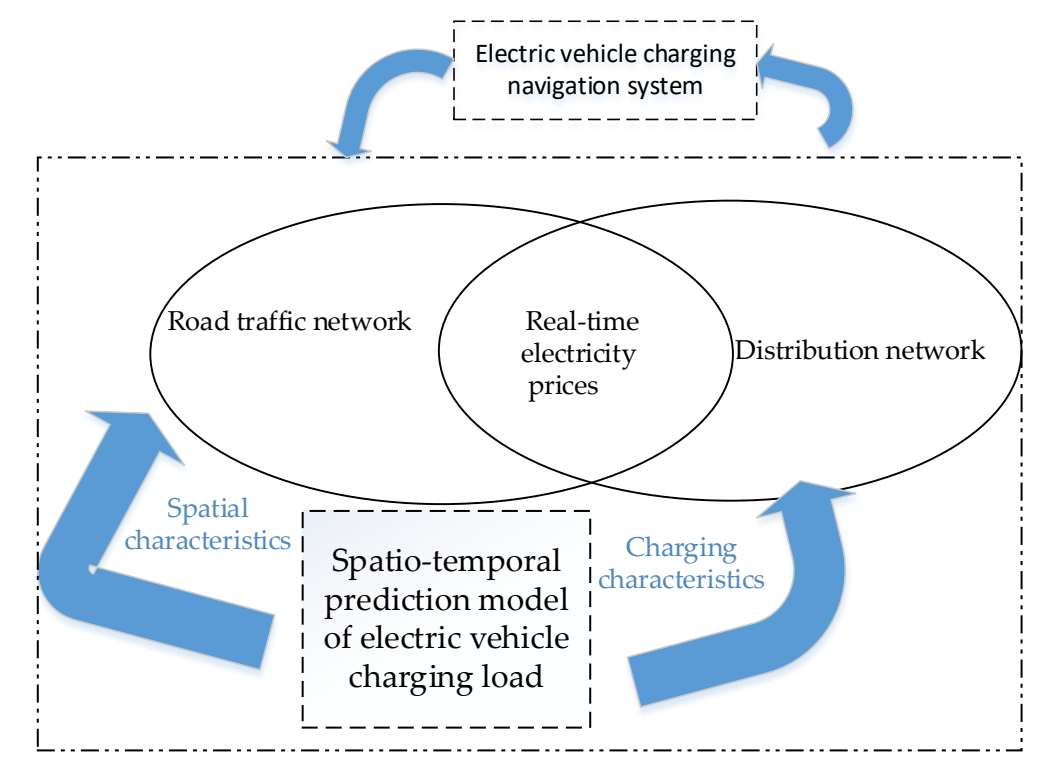

Figure 1. Relationship between subsystems.

\subsubsection{Road Topology}

Let $R n=\left(N_{d}, S, L, V, Q, K\right)$, and represent the road network.

$N_{d}$ is the set of road crossing nodes in the road network system, and the topological structure of the traffic network is shown by taking Figure 2 as an example. 


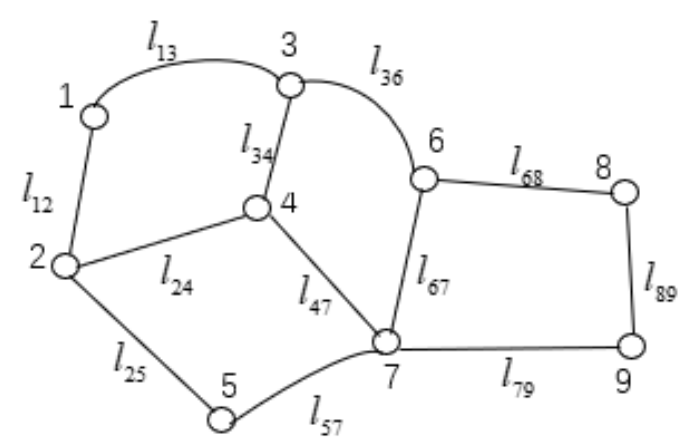

Figure 2. Traffic network topology diagram.

$S$ is the adjacency matrix set of road weight, which is used to describe the length of each road segment and the connection relationship between nodes:

$$
S(x, y)=\left\{\begin{array}{c}
l_{x y}, l_{x y} \neq 0, \text { Length of section }(x, y) \\
0, x=y \\
\inf , \text { Section }(x, y) \text { is not connected }
\end{array}\right.
$$

where $l_{x y}$ is the length of the link between adjacent nodes $x$ and $y$; inf represents infinity, that is, the two nodes are not adjacent. When the road network model is assumed to be a two-lane channel,

$$
S(x, y)=S(y, x)=l_{x y}, \forall x, y \in N, x \neq y
$$

According to the matrix $S$ generated in Figure 2, under the Dijkstra shortest path algorithm, the shortest path between nodes is obtained.

$$
S=\left[\begin{array}{ccccccccc}
0 & l_{12} & l_{13} & \text { inf } & \text { inf } & \text { inf } & \text { inf } & \text { inf } & \text { inf } \\
l_{21} & 0 & \text { inf } & l_{24} & l_{25} & \text { inf } & \text { inf } & \text { inf } & \text { inf } \\
l_{31} & \text { inf } & 0 & l_{34} & \text { inf } & l_{36} & \text { inf } & \text { inf } & \text { inf } \\
\text { inf } & l_{42} & l_{43} & 0 & \text { inf } & \text { inf } & l_{47} & \text { inf } & \text { inf } \\
\text { inf } & l_{52} & \text { inf } & \text { inf } & 0 & \text { inf } & l_{57} & \text { inf } & \text { inf } \\
\text { inf } & \text { inf } & l_{63} & \text { inf } & \text { inf } & 0 & l_{67} & l_{68} & \text { inf } \\
\text { inf } & \text { inf } & \text { inf } & l_{74} & l_{75} & l_{76} & 0 & \text { inf } & l_{79} \\
\text { inf } & \text { inf } & \text { inf } & \text { inf } & \text { inf } & l_{86} & \text { inf } & 0 & l_{89} \\
\text { inf } & \text { inf } & \text { inf } & \text { inf } & \text { inf } & \text { inf } & l_{97} & l_{98} & 0
\end{array}\right]
$$

\subsubsection{Speed-Density Model}

$L$ is the road grade of the road section. Divided into five road levels [18]. The traffic density is $K=N / L$, and the traffic flow is $Q=N / T$. Road speed is determined by both road grade and traffic flow (see Table 1).

Table 1. Road grade.

\begin{tabular}{ccc}
\hline Road Grade & $\boldsymbol{V m} / \mathbf{( k m} / \mathbf{h})$ & $Q /($ Traffic/h) \\
\hline 1 & 80 & 1200 \\
2 & 60 & 900 \\
3 & 50 & 660 \\
4 & 40 & 660 \\
5 & 30 & 540 \\
\hline
\end{tabular}


From the urban road traffic speed-density model [19], we can get:

$$
V=\left\{\begin{array}{l}
V_{m} \ln \left(K_{j} / K\right)(\text { Blocking flow }) \\
\left.V_{f} e^{-\left(K / K_{m}\right)} \text { (Free flow }\right)
\end{array}\right.
$$

Greenberg's logarithmic model and Underwood's exponential model are applicable to block flow and own flow, respectively. The curves of the two models intersect in the middle and the traffic density of the intersection is $K_{0}$. A logarithmic model is used for the obstructed flow (left of the intersection); an exponential model is used for the free flow (right of the intersection). Among them, $V_{m}$ is the zero-flow speed, $K_{m}$ is the optimal density at the maximum traffic volume, $\mathrm{Vf}$ is the ideal smooth speed, and $K_{j}$ is the maximum traffic density.

Available traffic flow formula:

$$
Q=K V=\left\{\begin{array}{l}
K V_{m} \ln \left(K_{j} / K\right), \text { (Blocking flow) } \\
K V_{f} e^{-\left(K_{j} / K_{m}\right)},(\text { Free flow })
\end{array}\right.
$$

Define the road congestion rate $\rho=1 / V$, which means road congestion.

\subsubsection{OD Analysis Method}

The survey of traffic start and end points is also called OD traffic volume survey. OD traffic volume refers to the amount of traffic travel between the starting and ending points. "O" comes from the English origin, which indicates the starting point of the line, and " $\mathrm{D}$ " comes from the English destination, which indicates the line's destination. OD survey results are usually expressed in a two-dimensional table, called OD matrix. The vehicle OD matrix is an important data in the prediction and analysis of electric vehicle traffic distribution. This paper adopts the OD back-calculation matrix based on the mathematical programming method [20], that is, to estimate the distribution of trips in the road network of the traffic flow of the road section. For a certain road network, two or more nodes are first matched as a start-end point pair. Assuming that the traffic allocation on the road sections at a time is $Y_{i}(i=1,2, \ldots, n), Y_{i}$ can be expressed as a linear combination of $X_{j}$ :

$$
Y_{i}=P_{i 1} X_{1}+P_{i 2} X_{2} \ldots+P_{i n} X_{n}
$$

$P_{i n}$ is expressed as the distribution ratio of the $n$-th starting and ending points in the travel distribution amount to the link $i$.

The traffic distribution of all road sections is as follows [20]:

$$
\left\{\begin{array}{l}
Y_{1}=P_{11} X_{1}+P_{12} X_{2} \ldots+P_{1 n} X_{n} \\
Y_{2}=P_{21} X_{1}+P_{22} X_{2} \ldots+P_{2 n} X_{n} \\
\cdots \\
Y_{m}=P_{m 1} X_{1}+P_{m 2} X_{2} \ldots+P_{m n} X_{n}
\end{array}\right.
$$

The OD probability matrix is:

$$
P X=Y
$$

By performing the inverse calculation on Equation (8), $X=P^{-1} Y$ is obtained, $X$ is the inverse OD travel matrix obtained by the inverse OD mode, and $Y$ is the road flow matrix distributed at the current time. Assume that the current number of road sections is $m$, and the number of pairs of start and endpoints is $n$.

Solve:

$$
\min f\left(X_{j}\right)=\sum_{i=1}^{m} S_{i}{ }^{2}=\sum_{i=1}^{m}\left(\sum_{j=1}^{n} P_{i j} X_{j}-Y_{i}{ }^{\prime}\right)^{2}
$$


Among them:

$$
S_{i}=Y_{i}-Y_{i}^{\prime}=\sum_{j=1}^{n} P_{i j} X_{j}-Y_{i}^{\prime}(j=1,2, \cdots n)
$$

$Y_{i}{ }^{\prime}$ is the actual road flow. The system of equations consisting of $m$ equations containing $n$ travel distributions can be reduced to a system of $n$-order equations with only one unknown quantity. Through the above mathematical programming method, a unique OD travel matrix $X$ is obtained. Electric vehicles can simulate their driving routes under the OD matrix.

\subsection{Real-time Electricity Price Model}

Electricity prices fluctuations and demand interact with each other. Charging stations set prices in a short time interval (10 $\mathrm{min})$. Charging cars grasp the real-time changes in electricity prices by interacting with charging stations. The price elasticity coefficient $e$ is a standardized quantity of the relationship between price and load change. Based on the customer satisfaction model ACSI [21], the expected satisfaction $\delta$ for electric vehicle charging is obtained, and the quantitative relationship [22] between the two is approximated by the revised price elasticity coefficient $e^{\prime}=e+\delta$.

\subsubsection{Load Elasticity Coefficient}

$$
e=(\partial p / \partial m)=\left(\Delta p_{s} / p_{s}\right) /\left(\Delta m_{t} / m_{t}\right)
$$

In the formula: $\Delta m$ and $\Delta p$ respectively represent the micro-increment of the electric vehicle charging load and the fluctuation of the electricity price; $s$ and $t$ represent the charging station number, and $s=t$ represents the elasticity of the electricity price of the electric vehicle charging load of the current charging station to the charging station (hereafter load elasticity), when $s \neq t$, it means the current price of the charging station is elastic to the price of other charging stations (cross-load elasticity); $s, t=0,1$, $2, \ldots, n$. The real-time electricity price elasticity matrix of the charging station is expressed as:

$$
E=\left[\begin{array}{ccc}
e_{11} & \cdots & e_{1 n} \\
\vdots & \ddots & \vdots \\
e_{n 1} & \cdots & e_{n n}
\end{array}\right]
$$

The dynamic demand response of the load and the real-time electricity price elasticity are less than approximately a first-order linear relationship, that is, the electricity price elasticity coefficient remains unchanged within each period time (10 min).

\subsubsection{Construction of Electric Vehicle Charging Satisfaction Model $\delta$}

Based on the ACSI model, $\delta$ takes into account the cost savings of electric vehicle charging, the three factors of arrival distance and charging queue time, which is defined as the weighting factor $f_{\text {cost }}$ of the electric vehicle charging, the distance factor $f_{\text {arrival }}$, and the queuing time factor $f_{\text {queue }}$. Value, the $\delta$ expression is as follows:

$$
\delta=a f_{\text {cost }}+b f_{\text {arrival }}^{2}+c f_{\text {queue }}^{2}
$$

In the formula: $a, b, c$ are weighting coefficients, $a+b+c=1, f_{\text {cost }}$ is the first-level indicator, $f_{\text {arrival }}$ and $f_{\text {queue }}$ are the second-level indicators.

The revised electricity price elasticity coefficient $e^{\prime}=e+\delta$, then the revised real-time electricity price elasticity matrix of the charging station is expressed as:

$$
E^{\prime}=\left[\begin{array}{ccc}
e_{11}^{\prime} & \cdots & e_{1 n}^{\prime} \\
\vdots & \ddots & \vdots \\
e_{n 1}^{\prime} & \cdots & e_{n n}^{\prime}
\end{array}\right]
$$


Get the response formula of real-time electricity price of each charging station:

$$
\left[\begin{array}{c}
p_{1}^{\prime} \\
p_{2}^{\prime} \\
\vdots \\
p_{n}^{\prime}
\end{array}\right]=\left[\begin{array}{llll}
p_{1} & & & \\
& p_{2} & & \\
& & \ddots & \\
& & & p_{n}
\end{array}\right] E^{\prime}\left[\begin{array}{c}
\Delta m_{1} / m_{1} \\
\Delta m_{2} / m_{2} \\
\vdots \\
\Delta m_{n} / m_{n}
\end{array}\right]+\left[\begin{array}{c}
p_{1} \\
p_{2} \\
\vdots \\
p_{n}
\end{array}\right]
$$

In the formula: $p_{1}, p_{2}, \ldots, p_{n}$ are real-time prices before the electricity price changes; $p_{1^{\prime}}, p_{2^{\prime}}, \ldots$, $p_{n}{ }^{\prime}$ are real-time electricity prices after changes.

\subsubsection{Economic Benefits of Electric Vehicle Charging}

Electric Vehicle Charging cost $Q_{0}$ before real-time electricity price:

$$
Q_{0}=p_{0} M_{0}
$$

At present, China implements a step price mechanism [23]. In this paper, a fixed price $p_{0}=0.65$ yuan $/ \mathrm{kWh}$ is set as the research basis for the real-time price, $M_{0}$ represents a total load of electric vehicle charging.

Electricity charges $Q_{1}$ for electric vehicles after real-time electricity prices:

$$
Q_{1}=\sum_{t=1}^{T} \sum_{i=1}^{n} p_{t i} m_{t i}
$$

Among them, $p_{t i}$ and $m_{t i}$ represent the real-time electricity price and charging a load of each charging station in each period, and $T$ is the total number of periods.

To meet the inequality constraints of electric vehicle charging profit:

$$
Q_{1}<Q_{0}
$$

\subsection{Distribution Network Model}

According to the general requirements of GB/T 29781-2013 electric vehicle charging stations, all charging stations must be connected to the power distribution network of a $10 / 0.4 \mathrm{kV}$ transformer, and four main substations (designated as four power supplies) according to the charging station scale requirements of this article point). Based on the commonly used IEEE57 standard distribution network model, as shown in Appendix A.

The distribution network model is used to verify the impact of electric vehicle charging behavior on the power grid. Node parameters and branch parameters of each distribution network are standard values. The distribution network capacity is 200 MVA, the line resistance and reactance are $0.15 \Omega / \mathrm{km}$, all branch are connected, and the switches are ignored. Based on the modified IEEE57-node distribution network model, the distribution network model is expressed as $G r i d=\left(G_{\text {Node }}, G_{\text {Branch }}, G_{\text {Generator }}\right)$.

$G_{\text {Node }}$ represents the node type, active power, reactive power, reference voltage, conductance, and susceptance of each node in the grid; $G_{B r a n c h}$ represents the impedance and transmission power among the nodes; $G_{\text {Generator }}$ represents the location, capacity of the power node, and other parameters. We then introduce three measurement parameters of average voltage offset rate, network loss rate and grid load standard deviation of the distribution network:

$$
\begin{gathered}
\Delta U \%=\left(U-U_{n}\right) / U_{n} \times 100 \% \\
N_{P}=\left(1-p_{c} / p_{r}\right) \times 100 \%
\end{gathered}
$$




$$
\sigma=\sqrt{\frac{1}{n} \sum_{i=1}^{n}\left(m_{i}-m_{z}\right)^{2}}
$$

In the formula: rated voltage $U_{n}$; actual voltage $U$; grid input power $P_{r}$; grid output power $P_{c}$; line average load $m_{z} ;$ line load $m_{i}$.

\section{Navigation Model of Electric Vehicle Charging Path in "Traffic-Price-Distribution" Mode}

\subsection{Electric Vehicle Charging Model}

\subsubsection{State Parameters of Electric Vehicles}

$E V=\left(V_{d p}, C_{l v}, D, V_{r p}, V_{i p}, V_{c p}, V_{p c k}, B_{p}\right)$ is used to represent the state parameters of electric vehicles. $V_{d p}$ represents the starting point of the electric vehicle entering the transportation network. Each node of the transportation network is randomly determined in MATLAB, which is the starting point of the vehicle's driving path.

$C_{l v}$ indicates the current position of the electric vehicle. When the electric vehicle is traveling at the planned route, the current position are recorded in real-time.

$D$ represents the distance to reach the destination of the electric vehicle. When the electric vehicle determines the starting point, it also randomly determines its endpoint in the transportation network.

$V_{r p}$ represents the current remaining battery power of the electric vehicle, and the remaining power of the electric vehicle is recorded in real-time during the driving process.

$V_{i p}$ represents the initial electric capacity of an electric vehicle. When an electric vehicle is put into the transportation network, it randomly determines its initial electric capacity of $60-80 \% B_{p}$.

$V_{c p v}$ is the charging power of electric vehicles. Due to the fast charging behavior of large-scale electric vehicles, it will have a significant impact on traffic congestion and power distribution loads, and the timeliness of the slow charging of electric vehicles is poor. This article assumes that electric vehicles choose the fast charging method, and the charging can be completed within $0.5-1 \mathrm{~h}$, and the charging power is $45-60 \mathrm{~kW}$.

$V_{p c k}$ is the power consumption per kilometer of electric vehicles, and $V_{p c k}=0.2(\mathrm{kWh}) / \mathrm{km}$.

$B_{p}$ is the battery power of the vehicle when fully charged, and the charging of the electric vehicle battery is completed when it is fully charged.

\subsubsection{Charging Conditions}

To determine whether to charge based on the current electric vehicle status:

(1) the electric vehicle residual power $V_{r p}$ forces the electric vehicle to charge when it is below $30 \%$ of the battery power $B_{p}[24]$;

(2) the remaining power of the electric vehicle cannot reach the designated destination.

Time estimate. For an electric vehicle that needs to be charged, its complete power change process:

$$
t=\left\{\begin{array}{l}
t_{1}=\sum_{1}\left(l_{i} / v_{i}\right), D-d_{1} V_{p c k} \leq 30 \% B_{p} \\
t_{2}=\sum_{2}\left(l_{i} / v_{i}\right) \\
t_{3}=t_{1}+t_{2}+T_{w} \\
t_{4}=t_{3}+t_{c}
\end{array}\right.
$$

In the formula, $t_{1}$ indicates the time that the electric vehicle has traveled from the departure to the current position; $t_{2}$ indicates the time required to reach the charging station when the current position of the electric vehicle meets the charging conditions; $T_{w}$ indicates the queue time of the electric vehicle at the charging station; $t_{c}=\left(B_{p}-V_{r p}\right) / V_{c p v}$ represents the time required for the electric vehicle battery to be fully charged. 


\subsection{Optimal Charging Path Navigation Strategy}

To plan the optimal charging path for an electric vehicle under this electric vehicle charging path navigation model, you first need to determine traffic network parameters such as road length, road flow and driving speed, and $E V$ parameters such as the starting point, initial power, and destination of the electric vehicle. For electric vehicles that require charging, it is necessary to plan their optimal charging path navigation; if there is no demand for electric vehicles, the electric vehicles will travel on the prescribed route and will not participate in charging. Due to the comprehensive consideration of the distance from the electric vehicle to each charging station, the time to reach the charging station, the charging time, the queuing time and the real-time charging cost, etc. The multi-objective optimization problem is converted into a single-objective optimization problem through the judgment matrix method [25]. The above problem is converted into the charging cost of the electric vehicle to the charging station (charging cost), and then the lowest charging path is recommended for the electric vehicle, that is, the optimal charging path navigation.

Objective function: Minimizing electric vehicle charging costs as the goal of optimal charging path navigation

Target expression: $\min ($ Charging $)=\min \left(d_{2}, t_{2}, t_{c}, Q_{t}, q_{i}{ }^{\prime}\right)$

The judgment matrix method makes a pairwise comparison based on the relative importance of each target and uses the judgment number $C_{i j}$ to indicate the importance of the target $F_{i}$ relative to the target $F_{j}$, that is:

$$
C_{i j}=\left\{\begin{array}{l}
1, F_{i} \text { is as important as } F_{j} \\
3, F_{i} \text { is slightly more important than } F_{j} \\
5, F_{i} \text { is obviously more important than } F_{j} \\
7, F_{i} \text { is relatively important than } F_{j} \\
9, F_{i} \text { is extremely important than } F_{j}
\end{array}\right.
$$

Use judgment numbers to form judgment matrix $M$, namely:

$$
M=\left[\begin{array}{ccc}
C_{11} & \cdots & C_{1 n} \\
\vdots & & \vdots \\
C_{n 1} & \cdots & C_{n n}
\end{array}\right]
$$

In the formula, $\mathrm{n}$ is the number of targets; $C_{i i}=1, C_{i j}=1 / C_{j i}, i, j=1,2, \ldots, n$.

According to the criterion matrix $M$, the importance of the target $F_{i}$ in the entire problem can be given by the geometric mean of $C_{i i}$, that is, $\pi=\left(\prod_{j=1}^{n} C_{i j}\right)^{1 / n}$; the target weights are:

$$
w_{i}=\pi_{i} / \sum_{j=1}^{n} \pi_{j}
$$

Aiming at this objective function, the distance $d_{2}$ from the electric vehicle to each charging station and the time $t_{2}$ to reach the charging station are considered as the most important and first considered level; the reservation queue time $Q_{T}$ and the real-time charging fee $q_{i}{ }^{\prime}$ are the second-level targets; the charging time $t_{\mathrm{c}}$ is a third level goal. Goals of the same level have the same importance, and the importance of goals of different levels decreases in order. Take $C_{12}=1, C_{13}=5, C_{14}=3, C_{15}=3$, $C_{23}=5, C_{24}=3, C_{25}=3, C_{34}=1 / 3, C_{35}=1 / 3, C_{45}=1$, and the judgment matrix is:

$$
M=[115331153315151131313133111313311]
$$


After determining the target weight, the multi-objective optimization problem of charging cost is transformed into a single-objective optimization problem, and the original objective function is transformed into:

$$
\begin{gathered}
\min (\text { Charging })=\min w_{1} d_{2}+w_{2} t_{2}+w_{3} t_{c}+w_{4} Q_{T}+w_{5} q_{i}{ }^{\prime} \\
\rightarrow 0.349 d_{2}+0.349 t_{2}+0.07 t_{c}+0.116 Q_{T}+0.116 q_{i}{ }^{\prime}
\end{gathered}
$$

It can be obtained that the optimal charging path of the electric vehicle is aimed at minimizing the charging cost under the comprehensive multi-factor situation.

\subsection{Electric Vehicle Charging Path Navigation System}

Taking into account the actual charging behavior habits of electric vehicle users, combined with real-time electricity price factors of charging stations, a new electric vehicle path navigation system is constructed, as shown in Figure 3. The traffic information required by the charging navigation system includes traffic network parameters such as road length, road flow and driving speed, and $E V$ parameters such as the starting point, initial power, and destination of the electric vehicle.

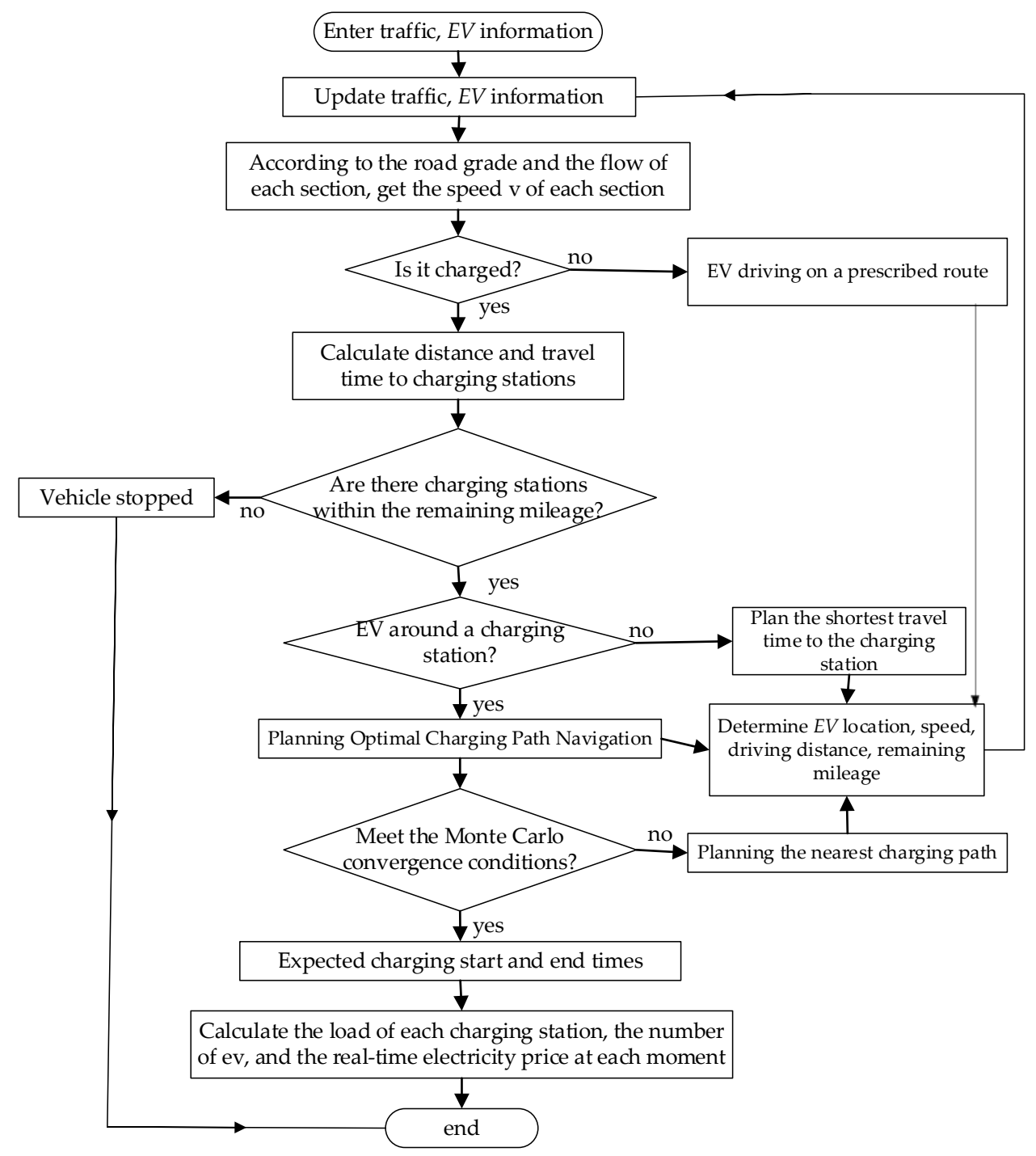

Figure 3. Charging path navigation diagram.

When the electric vehicle meets the charging conditions: the remaining electric power of the electric vehicle $V_{r p}$ is lower than $30 \%$ of the battery power $B_{p}$ or the remaining electric power of the electric 
vehicle cannot reach the designated destination. It is necessary to plan charging paths for electric vehicles in turn. Electric vehicles without charging requirements are driven on the route specified in the initial simulation. For electric vehicles that require charging, it is necessary to plan the charging path for each electric vehicle in order from the remaining power in ascending order. According to the Dijkstra shortest path algorithm, the travel distance and travel time from the current electric vehicle position to each charging station is calculated. First, we must determine whether an electric vehicle has a charging station in the driving range of its remaining power. If the electric vehicle cannot reach any charging station, the electric vehicle stops. When there is a charging station in the mileage range of the electric vehicle, it is determined that the EV is located around a charging station. Due to the actual charging behavior of electric vehicle users, if an electric vehicle is around a charging station, the charging station is the user's preferred target, then the charging path is the shortest charging path. If the electric vehicle is not located near any charging station, it navigates according to the optimal charging path to minimize the charging cost of the electric vehicle to obtain the optimal charging path of the electric vehicle. If the remaining electric power of the electric vehicle cannot reach the designated charging station according to the optimal charging path navigation, then within the range of the electric vehicle's driving range, the shortest path to each charging station is the minimum value which is the recommended charging path of the electric vehicle.

\section{Spatiotemporal Prediction Model of Electric Vehicle Charging Load}

\subsection{Frame Structure of Spatiotemporal Prediction of Charging Load}

The frame structure of spatiotemporal prediction of the electric vehicle charging load is shown in Figure 4.

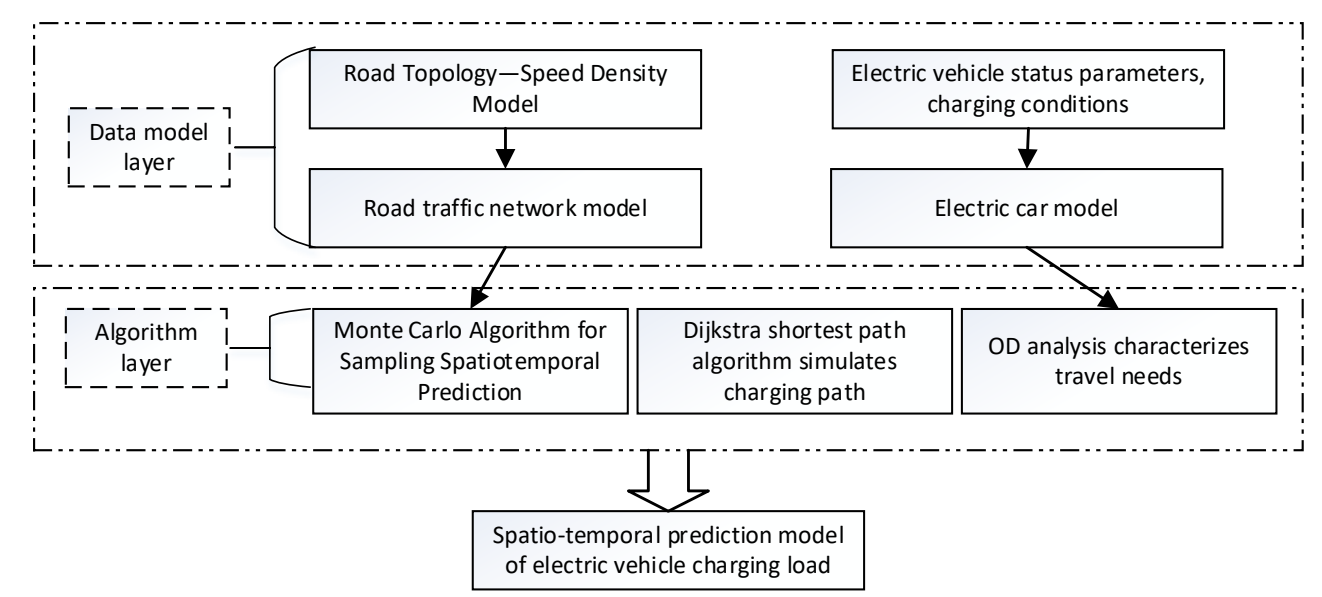

Figure 4. Space-time prediction frame structure of electric vehicle charging load.

In this framework, the data model layer contains a road traffic network model and an electric vehicle model based on the road topology and speed density model. In the prediction of electric vehicle charging load, the initial state parameters such as the remaining power, charging power, and simulation start position of the electric vehicle are randomly determined by the Monte Carlo algorithm, and according to the driving route planned by the electric vehicle in the OD matrix, the Dijkstra algorithm completes the navigation of the optimal charging path of electric vehicles. Finally, it traverses the spatiotemporal prediction information of all-electric vehicles under the Monte Carlo algorithm to obtain the spatiotemporal distribution data of the electric vehicle charging load and calculates the charging load $p(t)$ of each node of the distribution network. 


\subsection{Spatiotemporal Prediction Model of Charging Load}

The time-space prediction flowchart of the electric vehicle charging load is shown in Figure 5. In this paper, $T_{S}$ is used as the starting point of the simulation. The electric vehicle uses the Monte Carlo algorithm to sample the initial power, the starting point position, and the charging power. According to the electric vehicle state parameters and speed-density model, parameters such as the remaining electric power and position of the electric vehicle are updated. When the charging conditions are reached, the charging path is planned. Arrive at the charging station, record the remaining power of the electric vehicle, the time to start charging, the time required for charging, and the queuing order of the charging station to determine the space-time information of the charging load at the charging station. In a Monte Carlo simulation process, the space-time prediction of $\mathrm{N}$ electric vehicles is completed, and the charging load $P_{J}^{F}\left(t_{i}\right)$ of each electric vehicle is recorded, and the load is counted into the charging load $P(t)$ at each node of the distribution network.

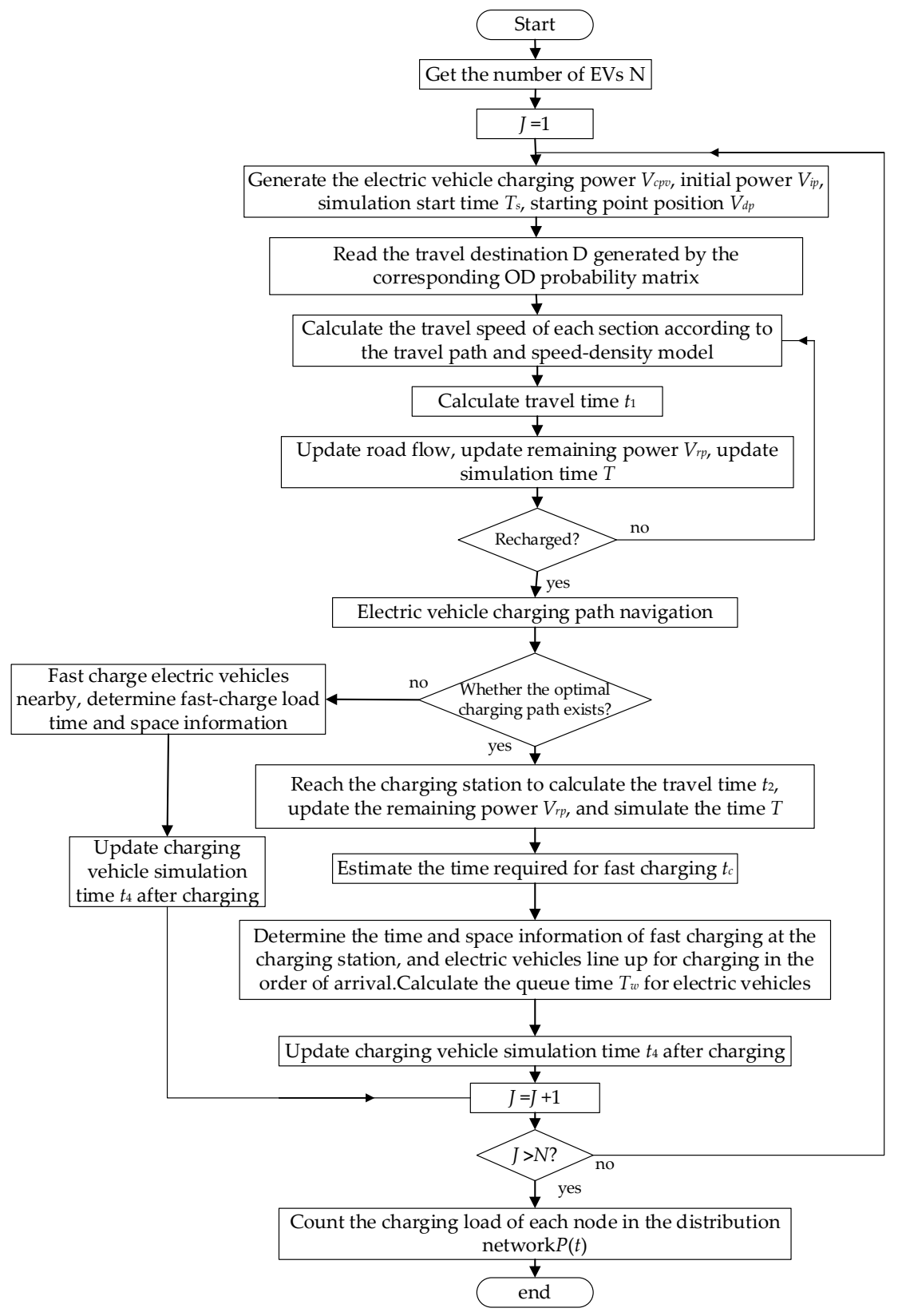

Figure 5. Flow chart of electric vehicle charging load space-time prediction. 
The travel destination $D$ generated by the electric vehicle corresponding to the OD matrix is read, and the travel speed of each road segment is calculated according to the travel path and the speed-density model. The travel time $t_{1}$, update the road flow, the remaining power $V_{r p}$, and update the simulation time $T$ are calculated. To determine whether the electric car has reached the charging conditions: the remaining electric power $V_{r p}$ of the electric car must be below $30 \%$ of the battery power $B_{p}$ or the remaining electric power of the electric car must be insufficient to reach the designated destination. When the electric vehicle reaches the charging station, the travel time $t_{2}$ is calculated, the remaining power $V_{r p}$ is updated, and the simulation time $T$ too. The time $T_{c}$ required for fast charging can be estimated. The time and space information of the fast charging of the current charging station are determined, and the electric vehicles are queued for charging in the order of arrival. The electric vehicle simulation time $t_{4}$ is updated at the end of charging. In a Monte Carlo simulation process, the space-time prediction of $\mathrm{N}$ electric vehicles is completed, and the charging load $P_{J}{ }^{F}\left(t_{i}\right)$ of each electric vehicle is recorded, and the load is counted into the charging load $P(t)$ of each node of the distribution network.

\subsection{Monte Carlo Simulation Convergence Conditions}

Based on the charging load spatiotemporal prediction model, with $10 \mathrm{~min}$ as a simulation interval, the Monte Carlo algorithm is used to obtain the simulation results of the spatiotemporal distribution of electric vehicles. The time $t_{c}$, charging load $p_{s}$, and charging start time $T_{s}$ of each electric vehicle is recorded. According to the situation that each charging station is connected to the distribution network node, the charging load is calculated into the corresponding distribution network node. At time $t_{i}$, the charging load $P^{F}\left(t_{i}\right)$ of node $F$ of the distribution network can be expressed as:

$$
P^{F}\left(t_{i}\right)=\sum_{n=1}^{m} P_{n}^{F}\left(t_{i}\right)
$$

In the formula, $m$ represents the number of electric vehicles connected to node $F$ at time $t_{i}$, and $P_{J}^{F}\left(t_{i}\right)$ represents the charging power of the $J$-th electric vehicle access node.

From this, the charging power matrix $L(t)$ of each node in the distribution network can be determined and then performing a power flow simulation analysis through MATPOWER. The flowchart is shown in Figure 6.

Monte Carlo convergence conditions: reach the set Monte Carlo simulation number $S$.

$$
\max \left|\frac{\sum_{i=1}^{S} L_{i}(t)}{S}-\frac{\sum_{i=1}^{S-1} L_{i}(t)}{S-1}\right|<\eta
$$

In the formula, $S$ is the maximum number of simulations, and $L_{i}(t)$ is the charging power matrix of the distribution network after the $i$-th time in Monte Carlo. $\eta$ is the Monte Carlo convergence accuracy, set to 0.01 ; the maximum number of simulations does not exceed 100 . 


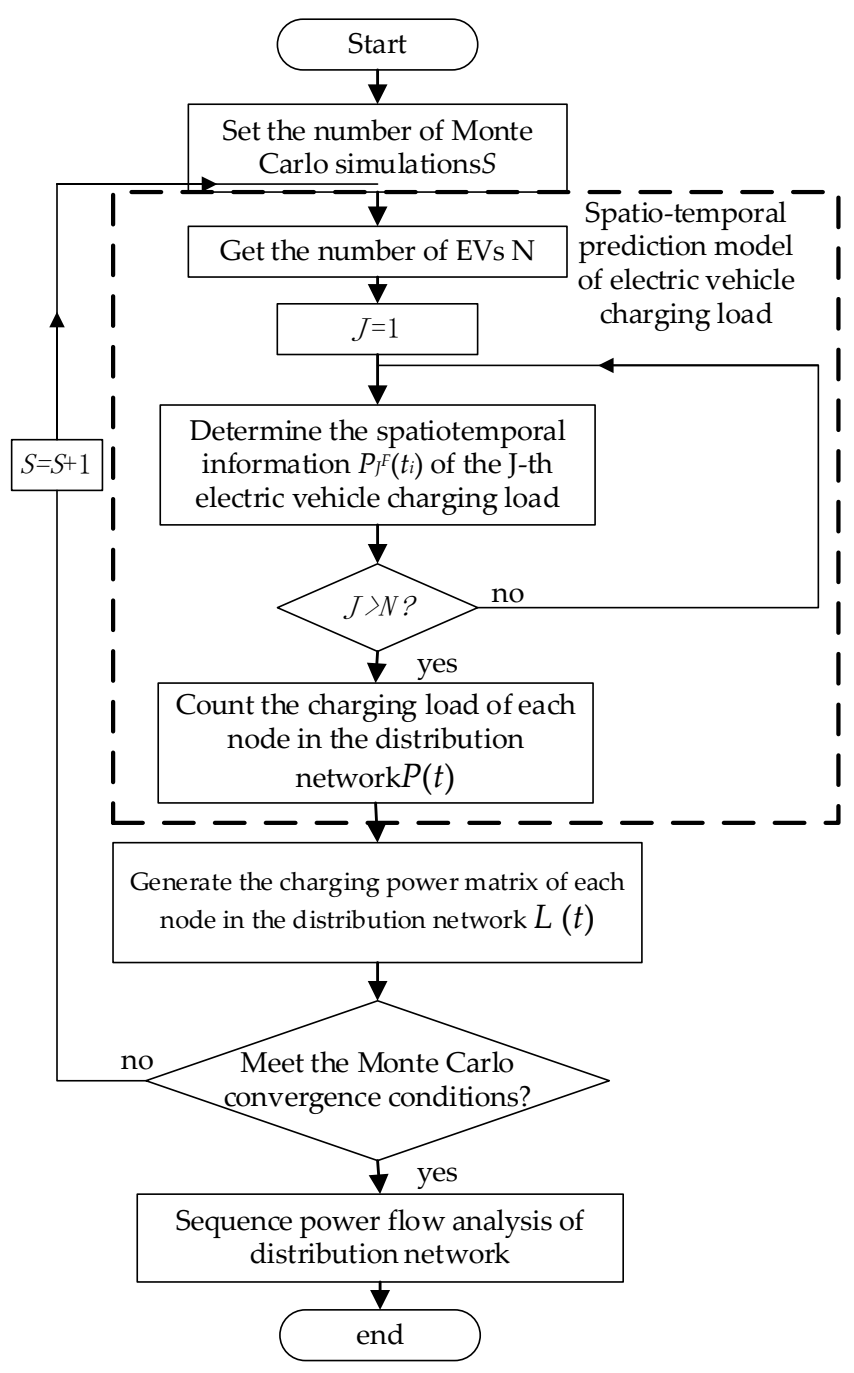

Figure 6. Monte Carlo simulation flow chart.

\section{Simulation Analysis}

\subsection{Simulation Condition Setting}

Figure 7 is a schematic diagram of traffic road levels, and the distribution of 11 charging station nodes is shown in the figure. The traffic network contains 107 roads and 61 road nodes, and the length information of each road is shown in Appendix B. The nodes of the road traffic network and the nodes of the power distribution network are geographically coupled. For details, see the attached Tables A2 and A3. This article uses Figure 7 as an example to perform a Monte Carlo simulation analysis of the electric vehicle charging path planning in this area.

In this area, a total of 40,000 EVs charging simulation processes were completed. The time period is set from 6:00 am to 6:00 pm. Large traffic flows are introduced between 9:00-10:00 and 14:00-15:00, forming a "double peak" situation of traffic flow; some electric vehicles are put into use at intervals of $10 \mathrm{~min}$.

First, it is determined whether the charging conditions are met based on the state of the electric vehicle that has been simulated. When the electric vehicle remaining power $V_{r p}$ is lower than $30 \%$ of the battery power $B_{p}$ or the electric vehicle remaining power cannot reach the designated destination, it is determined that the electric vehicle needs to perform a charging behavior and an optimal charging path is planned. If the charging conditions are not met, the electric vehicle travels on the route specified in the initial simulation, and the remaining power changes with time and changes according 
to the amount of power consumption. If the electric vehicle is around a certain charging station, it is charged the electric vehicle at the current charging station location. For example, when an electric vehicle is not near any charging station, its optimal path is planned by the optimal charging path navigation. If the electric vehicle cannot reach the optimal charging station location, the nearest charging path is planned.

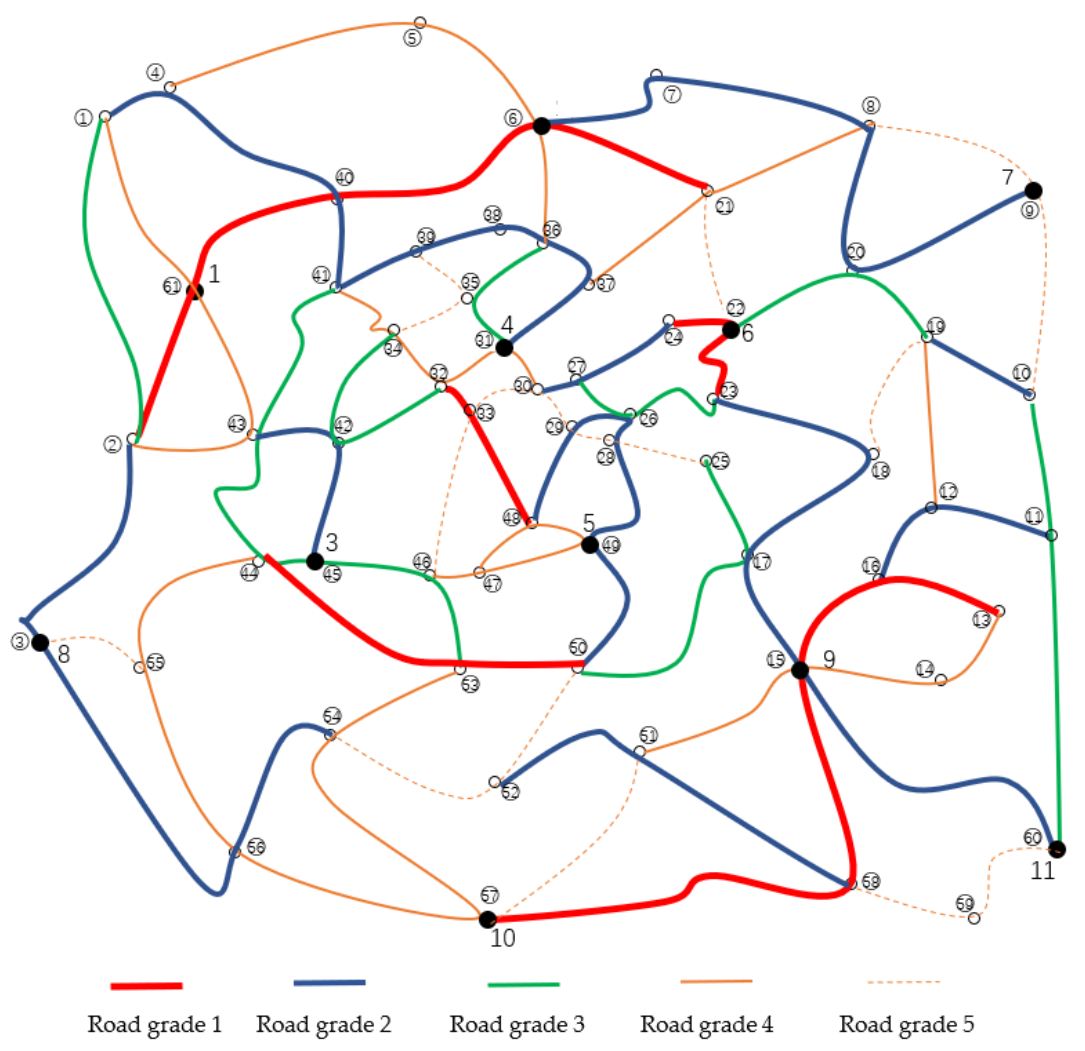

Figure 7. Traffic road grade diagram.

The charging navigation system aims at minimizing the charging cost of electric vehicles and plans an optimal charging path for each electric vehicle. When the electric vehicle reaches the endpoint specified at the charging station or simulation input, the simulation ends.

In the simulation process, only the road topology is used to replace the actual road to analysis. The electric vehicle travels at the same speed in the same section. The driving speed and the time to reach the charging station are determined by the traffic flow Equation (5) speed-density model.

\subsection{Simulation Analysis}

\subsubsection{Analysis of Temporal and Spatial Distribution Results of Charging Load}

Figure 8 shows the optimization situation of the number of electric vehicles in each charging station in planning the optimal charging path navigation under the "double peak" situation. Under the navigation planning of the optimal charging path for electric vehicles, the charging load distribution among charging stations is more evenly distributed, avoiding the situation of local charging station capacity overload. 


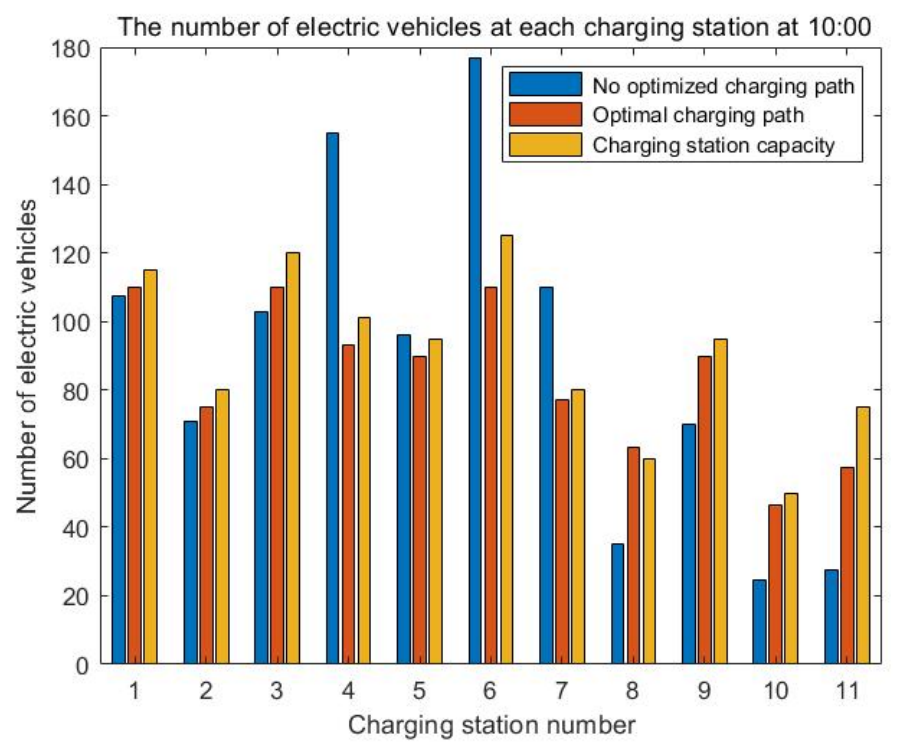

(a)

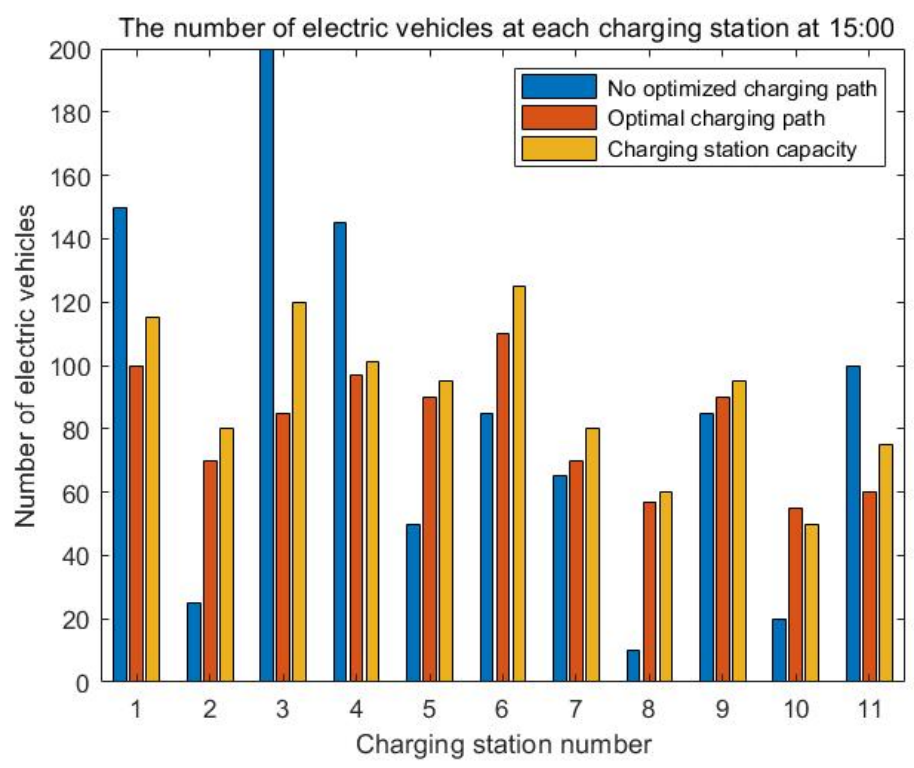

(b)

Figure 8. "Double peak" time charging station load comparison. (a) Comparison of the number of electric vehicles at each charging station at 10:00; (b) Comparison of the number of electric vehicles at each charging station at 15:00.

Electric vehicles are unevenly distributed in space, which may cause traffic congestion in the transportation network. The road congestion rate $\rho=1 / V$ is used to indicate the congestion of each road section. Under the guidance of the optimal charging path navigation, through the active allocation of space for electric vehicles, the problem of congestion on local roads is significantly alleviated. Figure 9 takes the average values of road congestion rate near charging stations at 10:00 and 15:00 respectively as examples. 


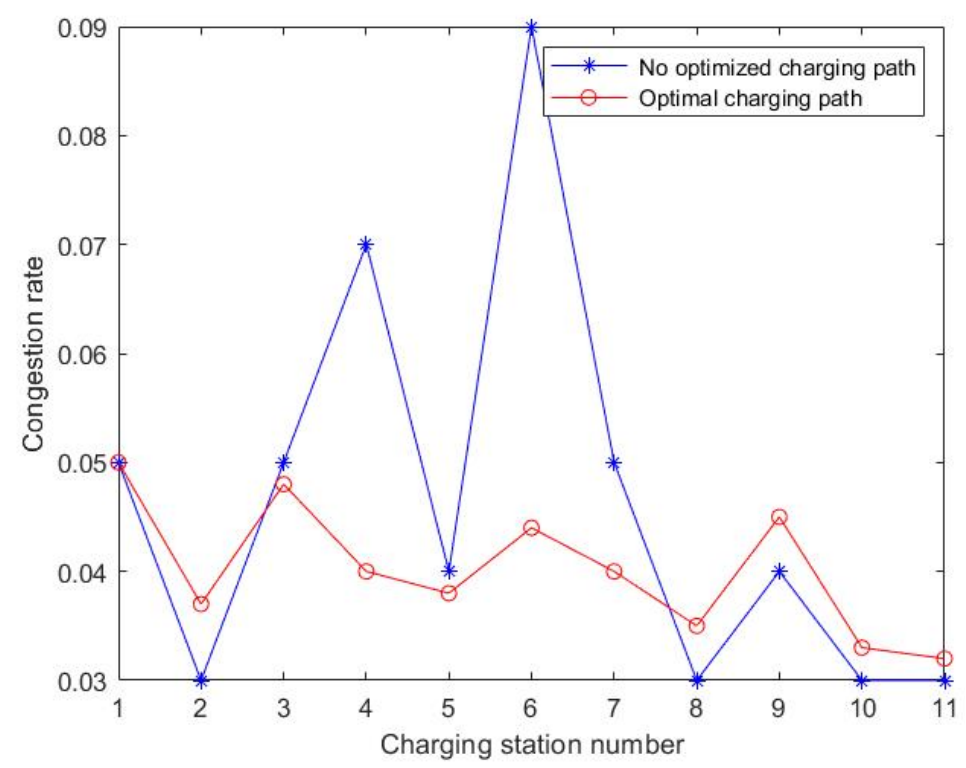

(a)

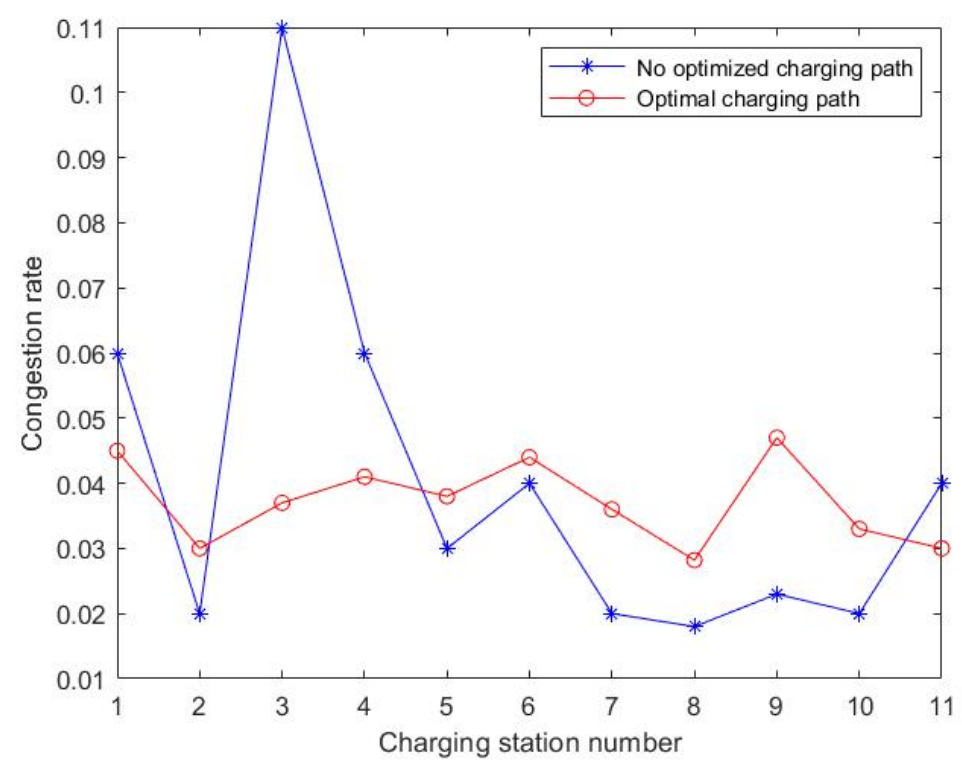

(b)

Figure 9. Comparison of road congestion rates near charging stations. (a) Average of road congestion rate near each charging station at 10:00; (b) Average of road congestion rate near each charging station at 15:00.

It can be concluded that there are differences in charging queuing time for electric vehicles under the conditions of optimal charging path navigation and no optimized charging strategy (see Table 2).

Table 2. Comparison of charging queue length of electric vehicle.

\begin{tabular}{ccc}
\hline Evaluation Index & Optimal Charging Path Navigation & No Optimized Charging Strategy \\
\hline EV number of queues & 307 & 2589 \\
EV average queue time & $15.4 \mathrm{~min}$ & $47.8 \mathrm{~min}$ \\
Longest queue time & $28.2 \mathrm{~min}$ & $71.9 \mathrm{~min}$ \\
\hline
\end{tabular}


Using the optimal charging path navigation, the charging queue time for electric vehicles has been significantly optimized. The longest queue time is $28.2 \mathrm{~min}$, which is much shorter than $71.9 \mathrm{~min}$ without the optimized charging strategy. The charging efficiency of electric vehicles has thus been significantly improved.

\subsubsection{Power Distribution Network Analysis}

Eleven charging stations are set up in the known model. To simplify the difference between the actual location of the charging station and the location of the distribution network node, it is assumed that the number of each charging station is in a coincident state with the distribution network node. Under the recommendation of optimal charging path navigation for electric vehicles, the Monte Carlo simulation is used to predict the charging time and space of electric vehicles, and the charging load changes at charging stations are shown in Figure 10. In order to make the results clearer, only the charging load changes of eight charging stations are given here.

It is not difficult to see that even in the dual-peak situation, the optimal charging path navigation can still make the charging load more reasonably distributed among the distribution network nodes. The charging load of each charging station is calculated to the corresponding distribution network node, and the power flow simulation analysis is performed to obtain the fluctuation difference caused by the charging behavior of the electric vehicle before and after optimization to the power grid.

Figures 11 and 12 are comparisons of the differences between the voltages of the 30-node distribution network caused by the charging load of electric vehicles in different scenarios.

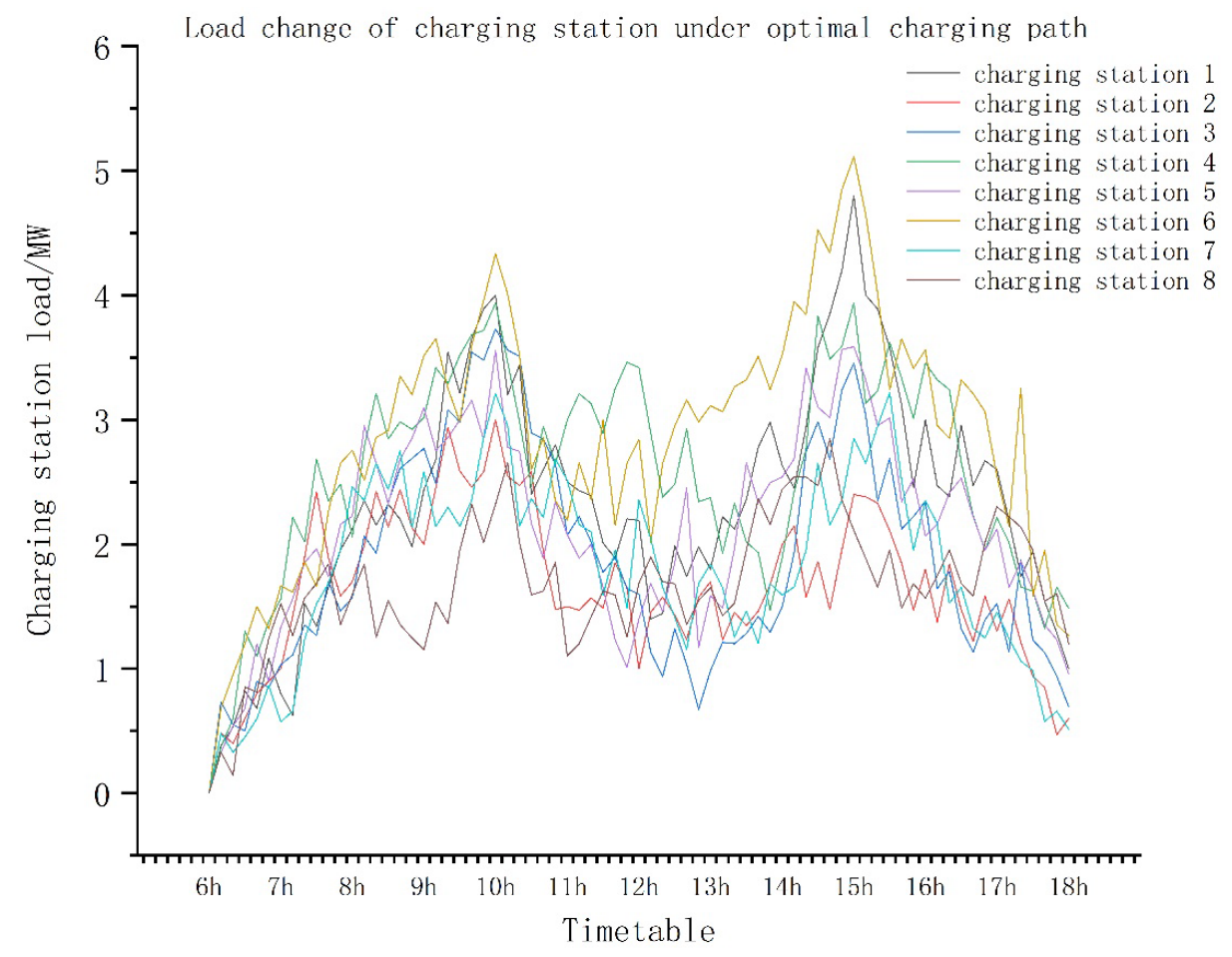

(a)

Figure 10. Cont. 
Load change of charging station without optimized charging path

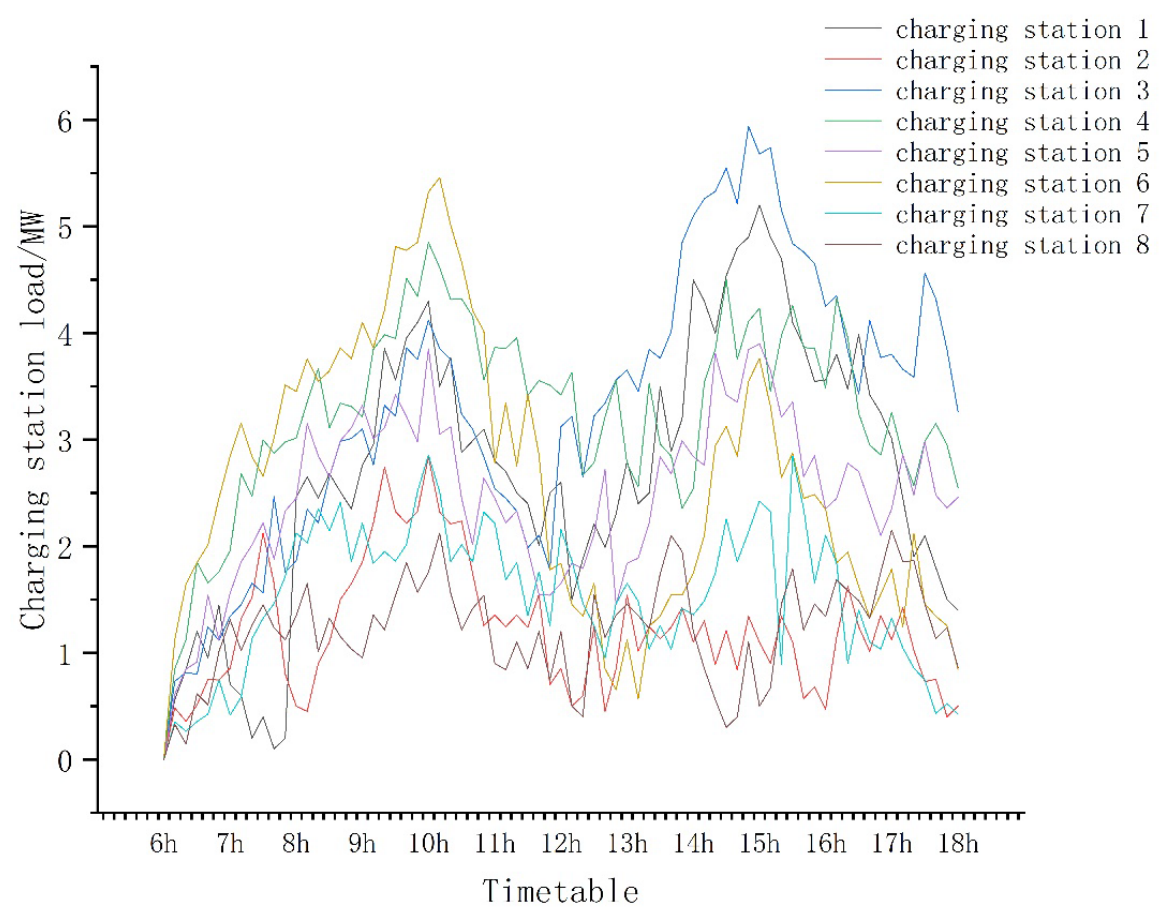

(b)

Figure 10. Eight charging stations' load changes with time. (a) Load change of charging station under navigation of optimal charging path; (b) load changes at charging stations without optimized charging strategy.

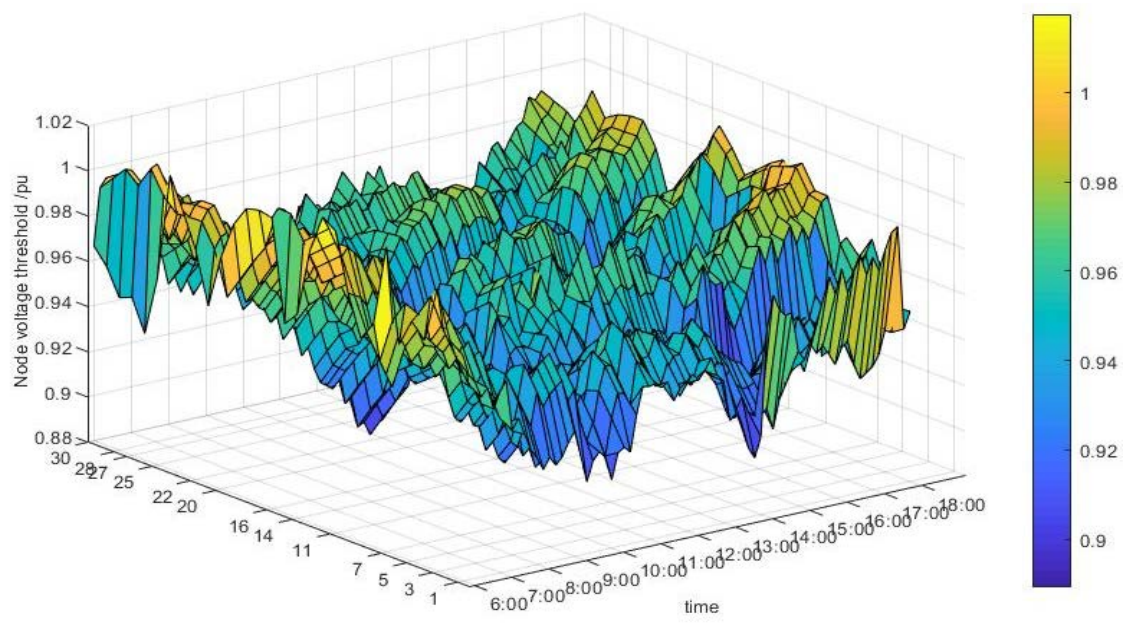

Distribution network node

(a)

Figure 11. Cont. 


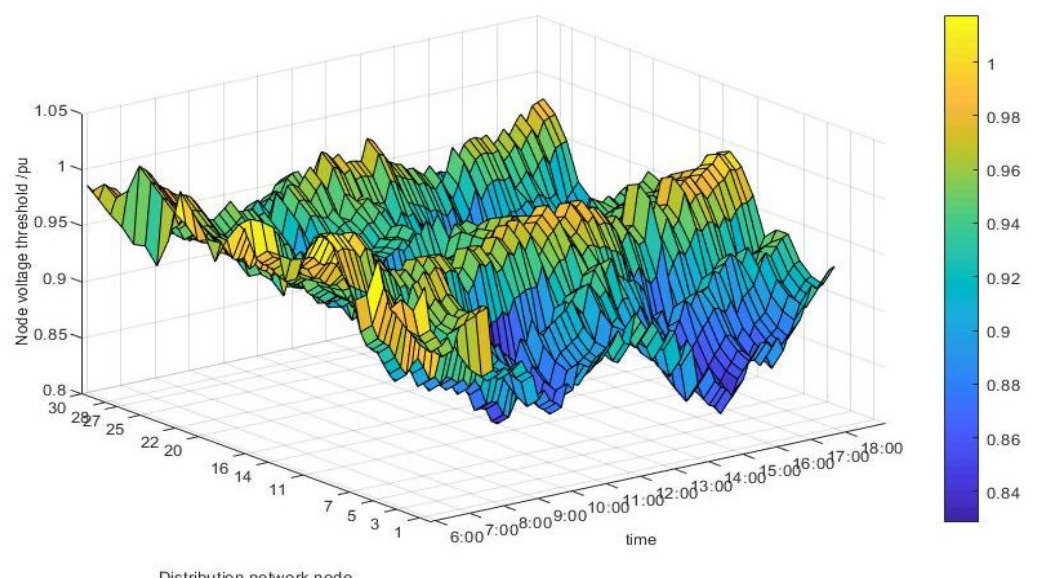

(b)

Figure 11. Distribution voltage distribution of 30-node distribution network in different scenarios. (a) 30-node distribution network voltage under the optimal charging path navigation; (b) voltage of 30-node distribution network without optimized charging strategy.

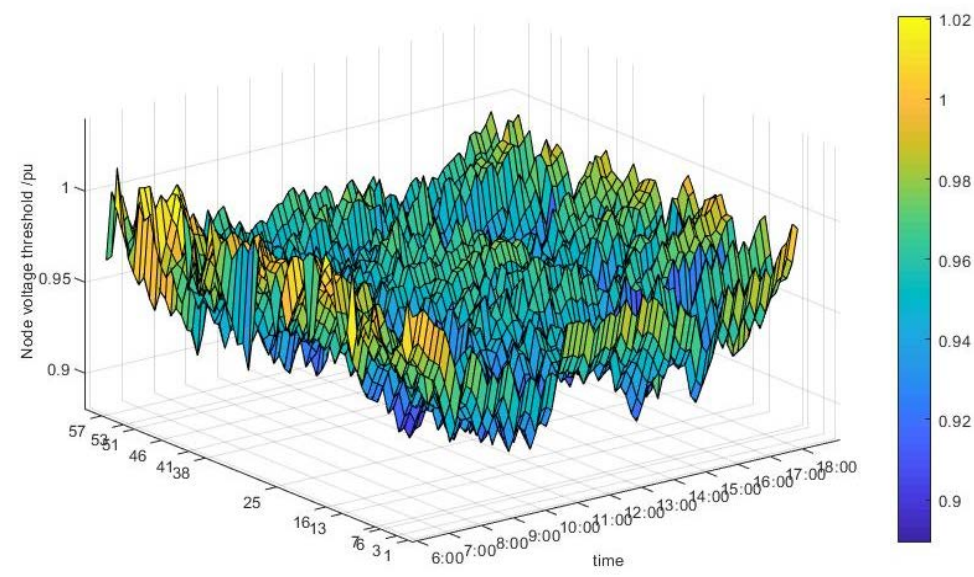

(a)

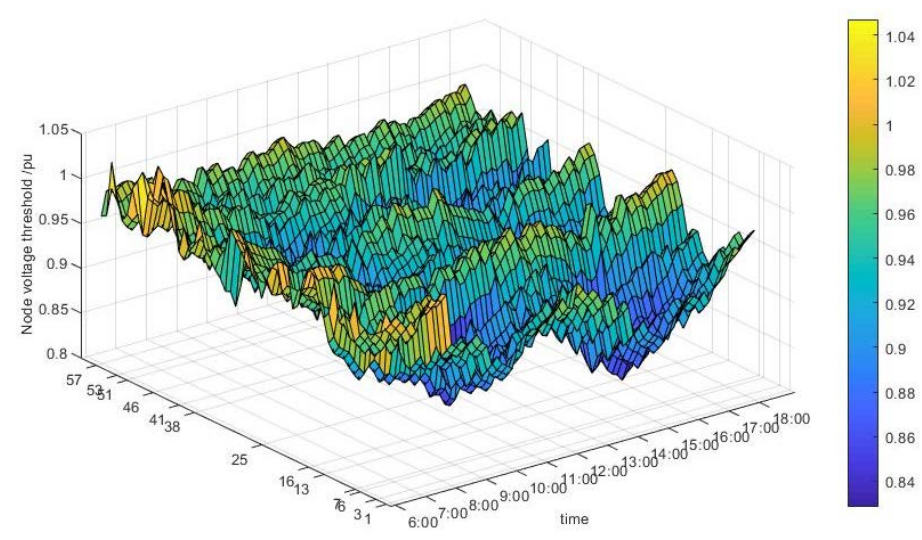

(b)

Figure 12. Distribution voltage distribution of 57-node distribution network in different scenarios. (a) 57-node distribution network voltage under the optimal charging path navigation; (b) voltage of 57-node distribution network without optimized charging strategy. 
In the power flow simulation results, the following measurement parameter indicators are obtained.

(1) Average voltage deviation rate:

Using the optimal charging path navigation, the average voltage deviation rate of the distribution network is smaller, and the node voltage has a better stability (see Table 3).

Table 3. Average voltage shift rate.

\begin{tabular}{ccc}
\hline \multirow{2}{*}{ Distribution Network } & \multicolumn{2}{c}{ Average Voltage Deviation (\%) } \\
\cline { 2 - 3 } & Optimal Charging Path Navigation & No Optimized Charging Strategy \\
\hline 30-node distribution network & 3.14 & 3.51 \\
57-node distribution network & 4.49 & 4.68 \\
\hline
\end{tabular}

(2) Network loss rate:

By adopting the optimal charging path navigation, the network loss rate of the distribution network has also decreased significantly, which has improved the economics of power grid operation (see Table 4).

Table 4. Network loss rate.

\begin{tabular}{ccc}
\hline \multirow{2}{*}{ Distribution Network } & \multicolumn{2}{c}{ Network Loss Rate (\%) } \\
\cline { 2 - 3 } & Optimal Charging Path Navigation & No Optimized Charging Strategy \\
\hline 30-node distribution network & 2.82 & 3.19 \\
57-node distribution network & 3.77 & 3.96 \\
\hline
\end{tabular}

(3) Standard deviation of grid load:

Using the optimal charging path navigation can make the electric vehicle load more reasonably distributed and avoid the situation of partial load overload.

Obviously, compared with the non-optimized charging strategy and the optimal charging path navigation strategy, the impact of electric vehicle charging behavior on the distribution network is significantly reduced (see Table 5). Among them, the overall performance improvement of the 30-node distribution network is more obvious, and the performance of the 57-node distribution network has also improved.

Table 5. Load standard deviation.

\begin{tabular}{ccc}
\hline \multirow{2}{*}{ Distribution Network } & \multicolumn{2}{c}{ Standard Deviation of Load } \\
\cline { 2 - 3 } & Optimal Charging Path Navigation & No Optimized Charging Strategy \\
\hline 30-node distribution network & 0.427 & 1.254 \\
57-node distribution network & 0.786 & 0.985 \\
\hline
\end{tabular}

\subsubsection{Analysis of Charging Convenience for Electric Vehicle Users}

According to the dynamic demand response of the electric vehicle load between charging stations, the elastic response of real-time electricity price is obtained.

If we take the real-time electricity price response of the No. 6 charging station shown in Figure 13 as an example, it is known that the fixed electricity price $p_{0}$ is $0.65 \mathrm{yuan} / \mathrm{kWh}$. The charging cost of electric vehicle users at No. 6 charging station without an optimized charging strategy is 105,731.34 yuan, and the charging cost under the real-time electricity price of the optimal charging path navigation is $134,193.97$ yuan. It can be concluded that during the entire charging process of all electric vehicles that need to be charged, the total charging cost under the real-time electricity price of the optimal charging path navigation is $Q_{1}=917,304.71$ yuan, and the total charging cost without the optimized charging strategy is $Q_{0}=1,042,968.19$ yuan, which meets the constraint condition $Q_{1}<Q_{0}$ (18). Obviously, 
large-scale electric vehicle charging according to the navigation planning charging path can help electric vehicle users to better save charging costs.

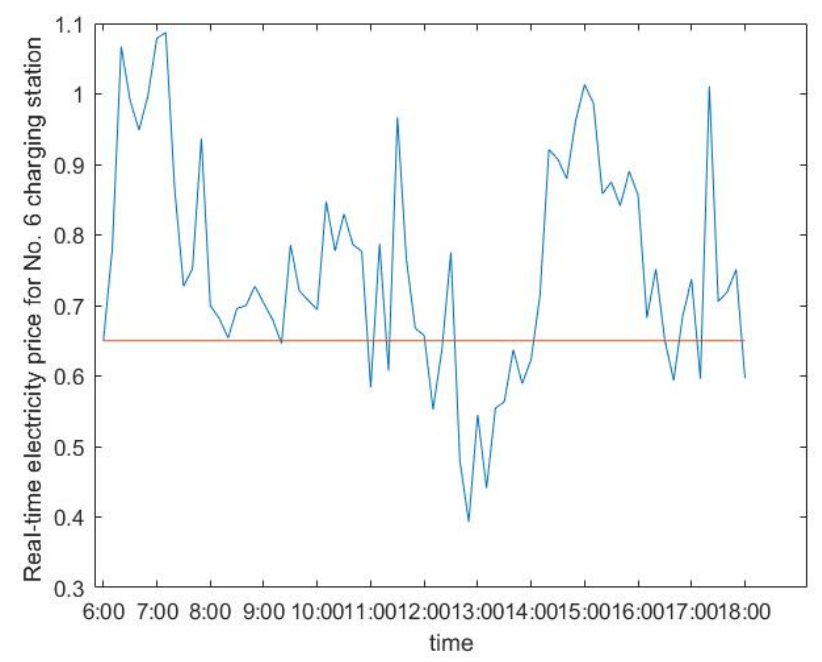

Figure 13. Real-time electricity price of the No. 6 charging station.

\section{Conclusions}

This paper proposes an optimization strategy for planning the optimal charging path of electric vehicles based on the "road-price-net" model and constructs a new electric vehicle path navigation system. Based on the Dijkstra shortest path algorithm and Monte Carlo spatiotemporal prediction method, the charging simulation process for 40,000 electric vehicles is completed. Based on the comparison between the optimal charging path navigation and the non-optimized charging strategy proposed in this paper, the following conclusions are concluded:

(1) Adopting the optimal charging path navigation, you can more rationally plan the charging load among charging stations, avoid the phenomenon of partial load overloaded at the charging stations as much as possible, effectively improve the charging efficiency of electric vehicles, and greatly reduce the length of the charging queue. It has certain reference value for the expansion of the charging station and capacity adjustment.

(2) In the simulation of the "double peak" phenomenon of real traffic, this article adopts the optimal charging path navigation method, which can effectively alleviate the local traffic congestion caused by the accumulation of more electric vehicles at one charging station to improve the operation of the entire transportation network.

(3) Compared with the non-optimized charging strategy, the average voltage offset rate, network loss rate, and the standard deviation of the grid load are significantly reduced when the optimal charging path is adopted for navigation. Besides, the overall operating capacity of the distribution network has been improved, and the impact of the charging behavior of electric vehicles on the entire distribution network has been minimized.

(4) Adopting the optimal charging path navigation can more reasonably save charging costs for electric vehicle users, from the perspective of the immediate interests of electric vehicle users.

In the simulation of this paper, certain assumptions are made on the types of electric vehicles, charging paths, and actual traffic conditions. Based on the existing "vehicle-network-road" model and the concept of the spatiotemporal prediction of the charging load of electric vehicles, the impact of real-time electricity prices on the selection of electric vehicle charging paths was initially discussed to establish a more complete electric vehicle charging path navigation system based on the consideration of charging users. Therefore, in the future, the planning of electric vehicle charging paths will be comprehensively considered from the distribution of charging facilities, capacity allocation, and road traffic simulation. 
Author Contributions: All authors contributed to the research in this paper. Z.W. and H.W. conceived and designed the model. X.F. and P.M. provided the data. W.Y. analyzed the data. Z.D. and Z.Y. wrote the paper. All authors have read and agreed to the published version of the manuscript.

Funding: This work is supported by the National Natural Science Foundation of China (grant no. 51477099); Open Project Foundation of Key Laboratory of Control of Power Transmission and Conversion (SJTU), Ministry of Education (grant no.2016AB14).

Conflicts of Interest: The authors declare no conflict of interest.

\section{Appendix A}

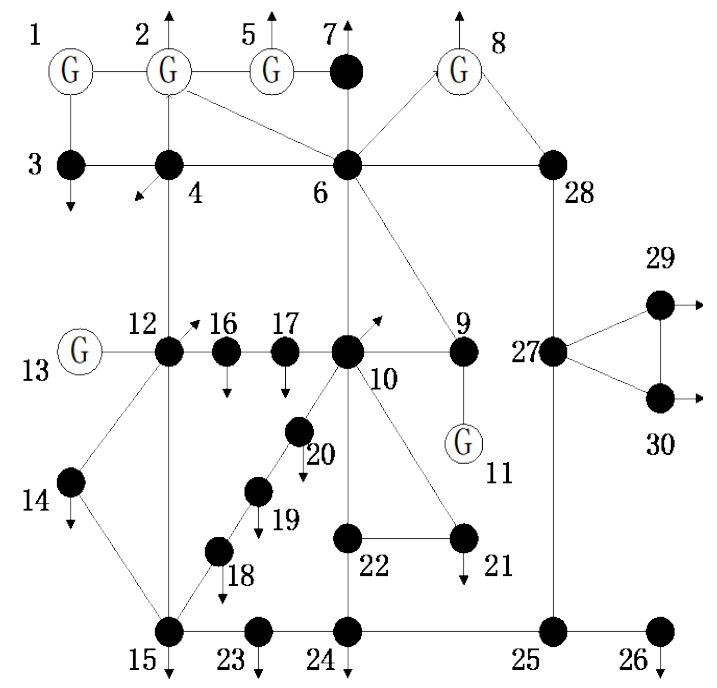

(a)

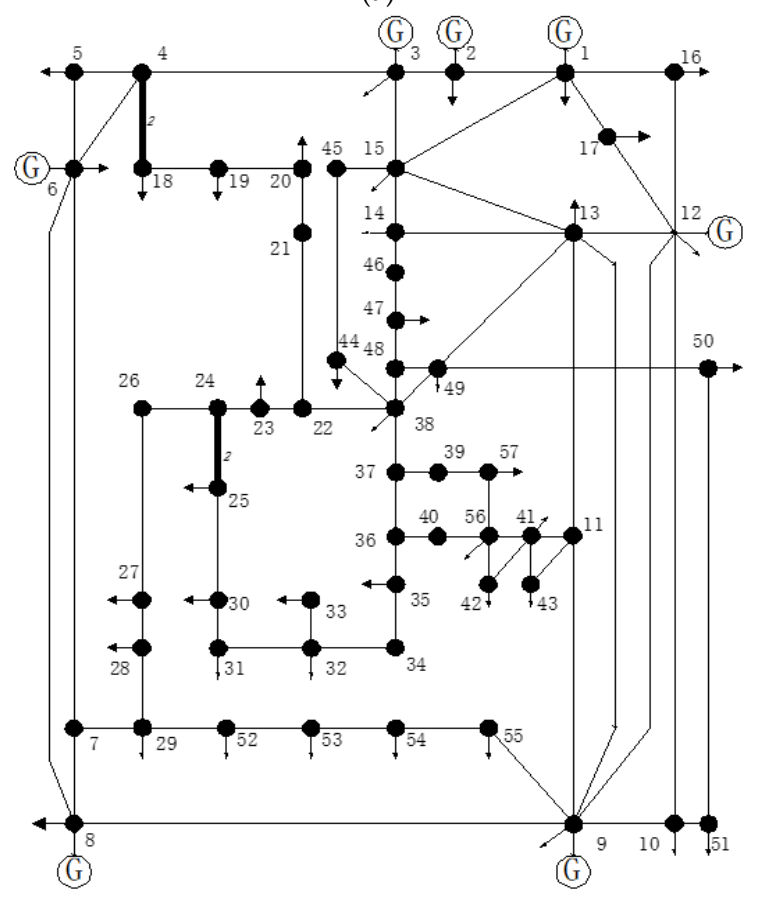

(b)

Figure A1. Topology of distribution systems. (a) 30-node distribution network; (b) 57 distribution network nodes. 
Table A1. Charging station number, 57-node distribution network node and transportation network node coupling correspondence table.

\begin{tabular}{ccccc}
\hline $\begin{array}{c}\text { Charging Station } \\
\text { Number }\end{array}$ & $\begin{array}{c}\text { Transportation } \\
\text { Network Node }\end{array}$ & $\begin{array}{c}\text { Distribution } \\
\text { Network Node }\end{array}$ & $\begin{array}{c}\text { Distribution } \\
\text { Network Node }\end{array}$ & $\begin{array}{c}\text { Power Point } \\
\text { Power (Kw) }\end{array}$ \\
\hline 1 & 61 & 6 & 1 & $129.9 \angle-7.12^{\circ}$ \\
2 & 6 & 3 & 3 & $40.0125<-1.432^{\circ}$ \\
3 & 45 & 25 & 8 & $454.265 \angle 7.857^{\circ}$ \\
4 & 31 & 46 & 12 & $335.5775 \angle 22.515^{\circ}$ \\
5 & 49 & 38 & - & - \\
6 & 22 & 13 & - & - \\
7 & 9 & 16 & - & - \\
8 & 3 & 7 & - & - \\
9 & 15 & 41 & - & - \\
10 & 57 & 53 & - & - \\
11 & 60 & 51 & & - \\
\hline
\end{tabular}

Table A2. Charging station number, 30-node distribution network node and transportation network node coupling correspondence table.

\begin{tabular}{ccccc}
\hline $\begin{array}{c}\text { Charging Station } \\
\text { Number }\end{array}$ & $\begin{array}{c}\text { Transportation } \\
\text { Network Node }\end{array}$ & $\begin{array}{c}\text { Distribution } \\
\text { Network Node }\end{array}$ & $\begin{array}{c}\text { Distribution } \\
\text { Network Node }\end{array}$ & $\begin{array}{c}\text { Power Point } \\
\text { Power (kW) }\end{array}$ \\
\hline 1 & 61 & 16 & 1 & $260.492 \angle 3.521^{\circ}$ \\
2 & 6 & 3 & 2 & $63.031 \angle 51.34^{\circ}$ \\
3 & 45 & 20 & 5 & $37 \angle 90^{\circ}$ \\
4 & 31 & 5 & 8 & $37.3 \angle 90^{\circ}$ \\
5 & 49 & 22 & 11 & $16.2 \angle 90^{\circ}$ \\
6 & 22 & 7 & - & - \\
7 & 9 & 25 & - & - \\
8 & 3 & 11 & - & - \\
9 & 15 & 27 & - & - \\
10 & 57 & 14 & - & - \\
11 & 60 & 28 & - & \\
\hline
\end{tabular}

\section{Appendix B}

Table A3. Network segment length table.

\begin{tabular}{cccccc}
\hline Road Section & Link Length $\mathbf{( k m )}$ & Road Section & Link Length $\mathbf{( k m )}$ & Road Section & Link Length $\mathbf{( k m )}$ \\
\hline$\{1,2\}$ & 6.52 & $\{17,18\}$ & 3.52 & $\{35,36\}$ & 2.61 \\
$\{1,61\}$ & 4.21 & $\{17,25\}$ & 2.38 & $\{35,39\}$ & 2.35 \\
$\{1,4\}$ & 2.16 & $\{17,50\}$ & 4.61 & $\{36,37\}$ & 2.56 \\
$\{2,3\}$ & 4.85 & $\{18,19\}$ & 3.57 & $\{36,38\}$ & 1.86 \\
$\{2,43\}$ & 2.51 & $\{18,23\}$ & 3.84 & $\{38,39\}$ & 2.23 \\
$\{2,61\}$ & 2.98 & $\{19,20\}$ & 3.21 & $\{39,41\}$ & 2.63 \\
$\{3,56\}$ & 7.84 & $\{20,22\}$ & 3.57 & $\{40,41\}$ & 2.75 \\
$\{3,55\}$ & 2.23 & $\{21,37\}$ & 3.48 & $\{41,43\}$ & 4.59 \\
$\{4,40\}$ & 5.16 & $\{21,22\}$ & 2.95 & $\{42,43\}$ & 2.49 \\
$\{4,5\}$ & 5.94 & $\{22,23\}$ & 2.15 & $\{42,45\}$ & 3.41 \\
$\{5,6\}$ & 2.84 & $\{22,24\}$ & 1.84 & $\{43,61\}$ & 3.38 \\
$\{6,40\}$ & 4.16 & $\{23,26\}$ & 2.54 & $\{43,44\}$ & 3.49 \\
$\{6,36\}$ & 3.154. & $\{24,27\}$ & 2.95 & $\{44,45\}$ & 1.42 \\
$\{6,7\}$ & 4.09 & $\{25,28\}$ & 2.46 & $\{45,46\}$ & 2.83 \\
$\{6,21\}$ & 4.59 & $\{26,27\}$ & 1.94 & $\{46,47\}$ & 1.92 \\
$\{7,8\}$ & 5.13 & $\{26,28\}$ & 1.26 & $\{46,53\}$ & 2.82 \\
\hline
\end{tabular}


Table A3. Cont.

\begin{tabular}{cccccc}
\hline Road Section & Link Length $\mathbf{( k m )}$ & Road Section & Link Length $\mathbf{( k m )}$ & Road Section & Link Length $(\mathbf{k m})$ \\
\hline$\{8,21\}$ & 4.59 & $\{26,29\}$ & 1.87 & $\{47,48\}$ & 2.4 \\
$\{8,20\}$ & 4.06 & $\{27,30\}$ & 1.59 & $\{47,49\}$ & 2.99 \\
$\{8,9\}$ & 4.35 & $\{28,29\}$ & 1.26 & $\{49,50\}$ & 4.21 \\
$\{9,20\}$ & 4.86 & $\{28,49\}$ & 3.85 & $\{50,53\}$ & 2.79 \\
$\{9,10\}$ & 4.66 & $\{29,30\}$ & 1.62 & $\{50,52\}$ & 3.38 \\
$\{10,19\}$ & 2.87 & $\{29,48\}$ & 3.16 & $\{51,52\}$ & 4.62 \\
$\{10,11\}$ & 2.81 & $\{30,31\}$ & 1.92 & $\{51,57\}$ & 5.5 \\
$\{11,12\}$ & 2.74 & $\{30,33\}$ & 2.24 & $\{51,58\}$ & 6.04 \\
$\{11,60\}$ & 5.95 & $\{31,32\}$ & 2.89 & $\{52,54\}$ & 4.26 \\
$\{12,19\}$ & 3.41 & $\{31,35\}$ & 2.36 & $\{53,54\}$ & 3.56 \\
$\{12,16\}$ & 2.21 & $\{31,37\}$ & 3.28 & $\{54,56\}$ & 4.5 \\
$\{13,16\}$ & 2.78 & $\{32,33\}$ & 1.68 & $\{54,57\}$ & 5.78 \\
$\{13,14\}$ & 2.01 & $\{32,34\}$ & 2.06 & $\{55,56\}$ & 5.74 \\
$\{14,15\}$ & 2.21 & $\{32,42\}$ & 3.54 & $\{56,57\}$ & 5.41 \\
$\{15,16\}$ & 2.74 & $\{33,46\}$ & 4.05 & $\{57,58\}$ & 6.81 \\
$\{15,17\}$ & 2.95 & $\{33,48\}$ & 3.54 & $\{58,59\}$ & 2.8 \\
$\{15,51\}$ & 3.63 & $\{34,41\}$ & 2.68 & $\{59,60\}$ & 3.21 \\
$\{15,58\}$ & 4.76 & $\{34,42\}$ & 3.46 & $\{1,40\}$ & 5.26 \\
$\{15,60\}$ & 6.48 & $\{34,35\}$ & 2.91 & $\{44,55\}$ & 3.81 \\
\hline
\end{tabular}

\section{References}

1. World Oil Outlook 2017. Available online: https://www.opec.org/opec_web/flipbook/WOO2017/WOO2017/ assets/common/downloads/WOO\%202017.pdf (accessed on 14 May 2019).

2. Cheng, Y.; Zhang, L. A survey of influence of electric vehicle charging on power grid. Power Syst. Technol. 2011, 35, 127-131.

3. Bunsen, T.; Cazzola, P.; Gorner, M.; Paoli, L.; Scheffer, S.; Schuitmaker, R.; Tattini, J.; Teter, J. Global EV Outlook 2018: Towards cross-Modal Electrification; International Energy Agency: Paris, France, 2018.

4. $\mathrm{Mu}, \mathrm{Y}$;; Wu, J.; Jenkins, N.; Jia, H.; Wang, C. A spatial-temporal model for grid impact analysis of plug-in electric vehicles. Appl. Energy 2014, 114, 456-465. [CrossRef]

5. Huang, H.; Chung, C.; Chan, K. Quasi-monte Caro based probabilistic small signal stability analysis for power systems with plug-in electric vehicle and wind power integration. IEEE Trans. Power Syst. 2013, 28, 3335-3343. [CrossRef]

6. Yilmaz, M.; Krein, P.T. Review of battery charger topologies, charging power levels, and infrastructure for plug-in electric and hybrid vehicles. IEEE Trans. Power Electron. 2013, 28, 2151-2169. [CrossRef]

7. Spitzer, M.; Schlund, J.; Apostolaki-Iosifidou, E.; Pruckner, M. Optimized Integration of Electric Vehicles in Low Voltage Distribution Grids. Energies 2019, 12, 4059. [CrossRef]

8. Jabalameli, N.; Su, X.; Deilami, S. An Online Coordinated Charging/Discharging Strategy of Plug-in Electric Vehicles in Unbalanced Active Distribution Networks with Ancillary Reactive Service in the Energy Market. Energies 2019, 12, 1350. [CrossRef]

9. Muratori, M. Impact of uncoordinated plug-in electric vehicle charging on residential power demand. Nat. Energy 2018, 3, 193-201. [CrossRef]

10. Diaz-Londono, C.; Colangelo, L.; Ruiz, F.; Patino, D.; Novara, C.; Chicco, G. Optimal Strategy to Exploit the Flexibility of an Electric Vehicle Charging Station. Energies 2019, 12, 3834. [CrossRef]

11. Yuichi, K.; Noboru, K.; Hirokazu, A. A Route Search Method for Electric Vehicles in Consideration of Range and Locations of Charging Stations. In Proceedings of the 2011 IEEE Intelligent Vehicles Symposium, Baden-Baden, Germany, 5-9 June 2011; p. 6.

12. Guo, Q.L.; Wang, Y. Research on architecture of ITS based smart charging guide system. In Proceedings of the IEEE Power and Energy Society General Meeting, Detroit, MI, USA, 24-29 July 2011.

13. Bayram, I.S.; Michailidis, G.; Devetsikiotis, M. Unsplittable load balancing in a network of charging stations under QoS guarantee. IEEE Trans. Smart Grid 2015, 6, 1292-1302. [CrossRef]

14. Xu, Z.W.; Su, W.C.; Hu, Z.C. A hierarchical framework for coordinated charging of plug-in electric vehicles in China. IEEE Trans. Smart Grid 2016, 7, 428-438. [CrossRef] 
15. Johnson, J.; Mashrur, C.; He, Y. Utilizing real-time information transferring potentials to vehicles to improve the fast-charging process in electric vehicles. Transp. Res. Part C Emerg. Technol. 2013, 26, 352-366. [CrossRef]

16. Yan, Y.Y.; Luo, Y.G.; Zhu, T. Optimal charging route recommendation method based on transportation and distribution information. Proc. CSEE 2015, 35, 310-318. (In Chinese)

17. Shao, Y.C.; Mu, Y.F.; Yu, X.D.; Dong, X.H.; Jia, H.J.; Wu, J.Z.; Zeng, W. A Spatial-temporal Charging Load Forecast and Impact Analysis Method for Distribution Network Using EVs-Traffic-Distribution Model. Proc. CSEE 2017, 37, 5207-5219. (In Chinese)

18. Xu, W.; Mohammad, S.; Chuanwen, J. Resilience Enhancement Strategies for Power Distribution Network Coupled with Urban Transportation System. IEEE Trans. Smart Grid 2018, 19, 4068-4079.

19. Hou, K.; Xu, X.D.; Jia, H.J. A reliability assessment approach for integrated transportation and electrical power systems incorporating electric vehicles. IEEE Trans. Smart Grid 2018, 9, 88-100. [CrossRef]

20. Mamei, M.; Bicocchi, N.; Lippi, M.; Mariani, S.; Zambonelli, F. Evaluating Origin-Destination Matrices Obtained from CDR Data. Sensors 2019, 19, 4470. [CrossRef] [PubMed]

21. American Customer Satisfaction Index. Customer Satisfaction Declines Across Entire Energy Utilities Sector, According to ACSI Data. Energy Weekly News, 19 March 2019.

22. Liu, C.; Feng, D.H.; Fang, C. A new pricing method for multi-solution of electricity price in real-time market. Proc. CSEE 2019, 1-14. (In Chinese)

23. China National Development and Reform Commission. Notice on Guiding Opinions on the Trial Implementation of Electricity Tariffs for Residents, 2011.11; Development and Reform Price [2011] No. 2617; National Development and Reform Commission: Beijing, China, 2011.

24. Luo, Y.G.; Yan, Y.Y.; Zhu, T. Intelligent electric vehicle optimal charging path planning method. Eng. Res. Eng. Interdiscip. Perspect. 2014, 6, 92-98.

25. Lu, T.; Tang, Y.; Cong, P.W.; Bo, B. Multi-objective coordination planning of distributed power supply and distribution network frame. Autom. Electr. Power Syst. 2013, 37, 139-145.

(C) 2020 by the authors. Licensee MDPI, Basel, Switzerland. This article is an open access article distributed under the terms and conditions of the Creative Commons Attribution (CC BY) license (http://creativecommons.org/licenses/by/4.0/). 